\title{
The role of cell lysis and matrix deposition in tumor growth modeling
}

\author{
R Santagiuliana ${ }^{{ }^{*}}$ (D) C Stigliano ${ }^{2}$, P Mascheroni ${ }^{1}$, M Ferrari ${ }^{3,4}$, P Decuzzi $i^{2,3,5}$ and B A Schrefler ${ }^{1,3}$
}

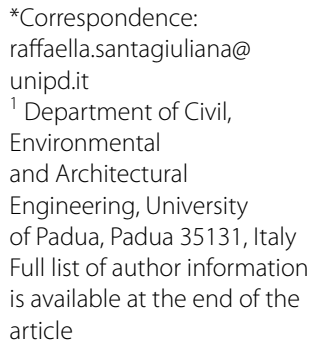

\begin{abstract}
Background: The multiphase model for tumor growth, proposed by the authors in previous works, is here enhanced. The original model includes a solid phase, the extracellular matrix (ECM) and three fluid phases: living and necrotic tumor cells (TCS), host cells (HCs), and the interstitial fluid (IF).

Methods: We introduce the mathematical model for deposition (remodeling) of ECM during the TCs growth, and lysis. Differently from the previous version of the model we take into account that TCs growing in vitro depose their own ECM not present at the beginning. The lysis re-transforms the necrotic cells into IF. The updated mathematical formulation is discretized by means of the finite element method and implemented in a general purpose code.

Results: First we reproduce new experimental data of multicellular tumor spheroid (MTS) growth in vitro. The free boundary conditions used in this simulation together with necrosis and lysis allow following the tumor growth curve up to the final steadystate. The second example, by comparing results of tumor growth in an ECM-free medium and in an ECM remodeling medium highlights how ECM deposition affects tumor growth. In an initially ECM-free medium the tumor is unobstructed and can proliferate more rapidly both without ECM and in case of ECM deposition. The third example shows the effect of lysis: it redirects some tumor cells toward the necrotic core of the MTS and produces outflow of the IF from the tumor mass.
\end{abstract}

Conclusions: The introduction of lysis and ECM deposition allows capturing different aspects of the avascular tumor growth not yet comprised in the original model: the MTS growth seems to be influenced by ECM deposition and the lysis seems to be a cause of an outflow of the IF from the tumor mass.

Keywords: Porous media mechanics, Multiphase system, Finite element method, Tumor spheroid, Lysis

\section{Background}

Much research went in the last two decades into the problem of tumor growth and drug delivery [1]. An extensive literature review [2] has shown that the most recent models for tumor growth are multicomponent models with or without diffuse interfaces among the constituents [3-10]. They all consider a malignant mass (tumor cells: TCs), host cells (HCs) and the interstitial fluid (IF) as homogeneous, viscous fluids and employ reactiondiffusion-advection equations for predicting the distribution and transport of nutrients. In general, these models contain limitations on the evolution of the volume fractions and

(c) 2015 Santagiuliana et al. This article is distributed under the terms of the Creative Commons Attribution 4.0 International License (http://creativecommons.org/licenses/by/4.0/), which permits unrestricted use, distribution, and reproduction in any medium, provided you give appropriate credit to the original author(s) and the source, provide a link to the Creative Commons license, and indicate if changes were made. 
on the velocities of the different cell populations and the IF is uncoupled from the rest. All models, with the exception of [10], disregard the contribution of the extracellular matrix (ECM). However experiments [11] clearly demonstrate that the tumor ECM is a source of resistance to the delivery of blood-borne drugs to cancer cells, especially for macromolecules and monoparticles [13]. The penetration of macromolecules correlates with ECM elasticity (deformability) and hydraulic conductivity (flow) [11, 13]. Further, TCs tend to deposit new ECM: as the mass of tumor cells increases; the ECM within the malignant tissue undergoes extensive rearrangements with increased deposition of collagen fibers, making the resulting tissue thicker and more difficult to penetrate [12, 13] as compared to host tissue.

We have developed a very general growth model for avascular tumors which is a multiphase flow model in a porous solid (ECM) [14-16]. The system is modeled at macroscopic scale making use of TCAT (thermodynamic constrained averaging theory). This model comprises TCs, which partition into living cells and necrotic cells, healthy cells (HCs), extracellular matrix (ECM) and interstitial fluid (IF). The ECM is a porous solid, while all other phases are treated as fluids. The IF transports chemical species such as nutrients, Tumor Angiogenic Factor (TFA), cytokines, etc. The interaction between the different compartments is fully taken into account through the stress tensor which involves the phase pressures, through the volumetric deformation appearing in the mass balance equations and through several constitutive equations. The flow aspects have been investigated in $[17,18]$. The model has been validated with respect to in vitro experiments from literature [19-21] and own experiments [18].

The role of the deformable ECM has been addressed in [22]. The adopted constitutive model is of the Green-elastic and elasto-visco-plastic type within a large strain approach. Truesdell objective stress measure is adopted together with the deformation rate tensor. The importance of including a deformable ECM in the model has been highlighted in an example of growth of a melanoma where different stiffness of the ECM leads to a different shape of the tumor. [22, 23].

We extend here the model to allow for observed ECM deposition and ECM remodeling which affect the rate of growth of tumor spheroids. Further we introduce lysis, i.e. the re-transformation of necrotic cells into IF.

The main objective is to explain some aspects of MTS growth, such as the possible causes of a more or less strong growth, outflow of the IF from the MTS, the final steady state in the growth curve, by means of the introduction of lysis and ECM deposition in the model. Also the effect of a moving boundary at the border of the MTS is investigated. It will be shown among other aspects that the combination of necrosis and lysis, together with a deformable ECM allows for reproducing outflow of IF from a tumor spheroid, as observed experimentally in [13].

The outline of the paper is the following: the updated mathematical formulation of the model allowing for ECM deposition and lysis are described in the "Methods" Section together with the necessary constitutive equations for fluids and the ECM. Numerical reproduction of experimental results of a multicellular tumor spheroid (MTS) growing in vitro and two examples of MTS growth are presented in Section "Results and discussion": the first of the two examples refers to the comparison between the growth of a MTS in an ECM deposited by TCs, in an ECM free culture medium and in a remodeling 
ECM scaffold; the second one shows the influence of lysis on the growth of a MTS. Conclusions and perspectives of the presented multiphase model complete the paper.

\section{Methods}

\section{The multiphase tumor growth model}

The updates introduced into our tumor growth model $[14,15,17,22]$ are now summarized. It is recalled that tumors are modeled at the macroscopic scale, being the domain of interest too large and the phase distributions too complex for modeling at the microscale. The TCAT approach [24-26] is used to transform known microscale relations to mathematically and physically consistent macroscale relations. These macroscale relations are adequate for describing the tumor behavior while filtering out high frequency spatial variability. The governing equations of the model are closed by introducing constitutive relations into the macroscale equations.

The multiphase system is comprised of the following phases: (1) tumor cells (TCs), which partition into living cells (LTC) and necrotic cells (NTC); (2) healthy cells (HCs); (3) extracellular matrix (ECM); and (4) interstitial fluid (IF); see [15, 17]. The ECM is a porous solid, while all other phases are fluids. The tumor cells may become necrotic upon exposure to low nutrient concentrations or excessive mechanical pressure [27]. The IF, transporting nutrients, is a mixture of water and biomolecules, such as nutrients, oxygen and waste products. In the following mass and momentum balance equations, $\alpha$ denotes a generic phase, $t$ the tumor cells (TCs), $h$ the healthy cells (HCs), $s$ the solid phase (ECM), and $l$ the interstitial fluid (IF).

The representative elementary volume of the multiphase system is depicted in the inset of Fig. 1 which shows an outline of the different stages of the modeling process.

A short overview of the mathematical model is given next; additional details are available in [22]. We use both vectorial and indicial notation where convenient.

\section{General governing equations}

The ECM is a deformable porous solid with porosity $\varepsilon$. The volume fraction of the solid phase is $\varepsilon^{s}=1-\varepsilon$. The other phases, tumor cells $\left(\varepsilon^{t}\right)$, healthy cells $\left(\varepsilon^{h}\right)$ and interstitial fluid $\left(\varepsilon^{l}\right)$, occupy the rest of the volume. The volume fractions for all phases add up to unity

$$
\varepsilon^{s}+\varepsilon^{h}+\varepsilon^{t}+\varepsilon^{l}=1
$$

The saturation degree of a fluid phase $\alpha$ is: $S^{\alpha}=\varepsilon^{\alpha} / \varepsilon$. Using porosity, $\varepsilon$, and volume fraction, $\varepsilon^{\alpha},(1)$ yields

$$
S^{h}+S^{t}+S^{l}=1
$$

As in the previous work [22], the primary variables of the model are: differential pressures $p^{h l}$ and $p^{t h}$, IF pressure $p^{l}$, nutrient mass fraction $\omega^{\overline{n l}}$, and displacement $u^{s}$ of solid phase (ECM).

The macroscopic mass and momentum balance equations of phases and species have been derived in [15] and their transformation to take into account of the differential pressures as primary variables has been obtained in [22]. The final form of the governing equations shown below are obtained from the general forms (see [22]) by introducing 


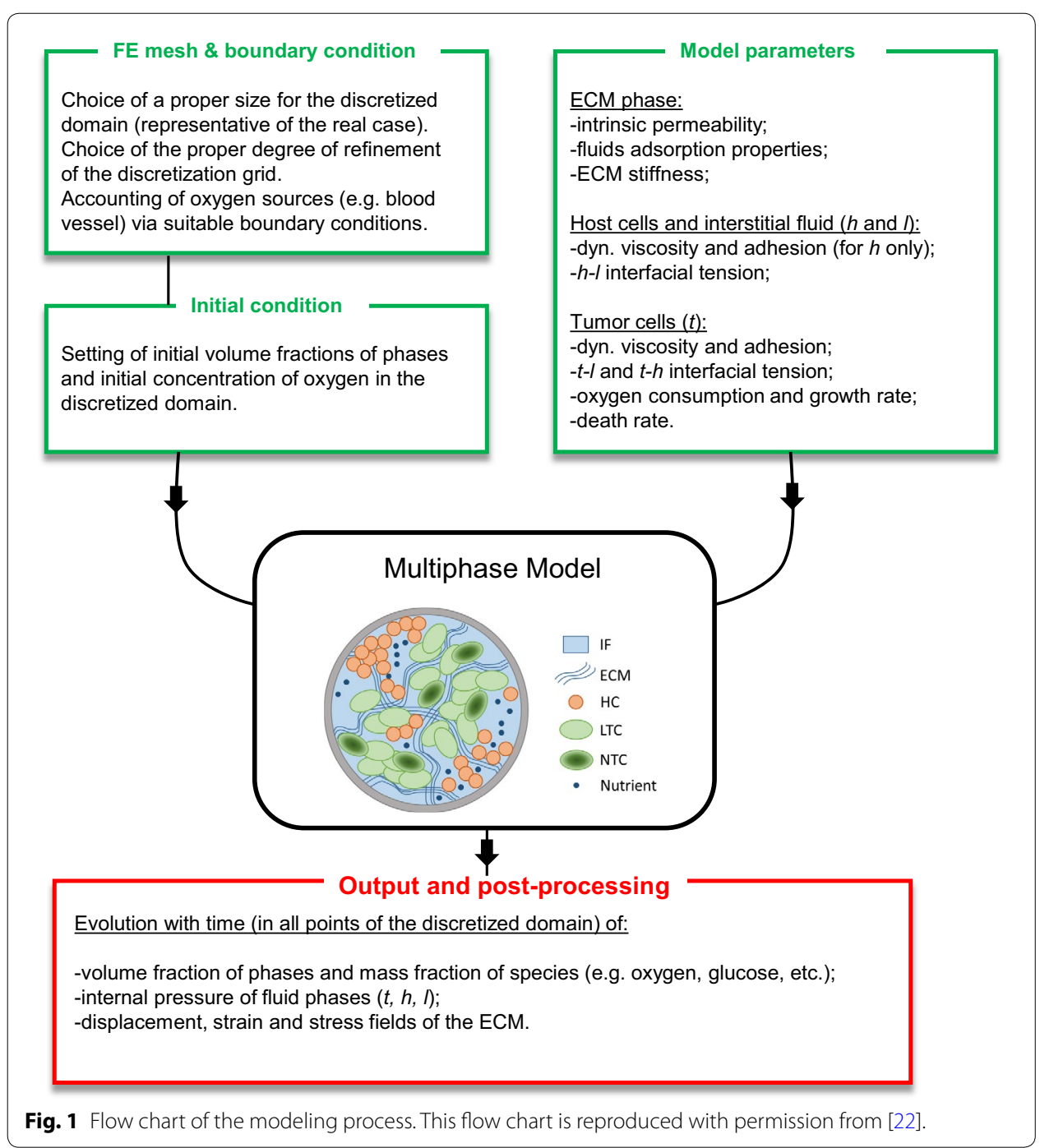

some simplifications and closure relationships (e.g., a Fickian type equation for diffusion of species, a generalized Darcy's equation for flow of the fluid phases, etc.).

New terms with respect to the previous works are present in the governing equations to introduce the two new aspects of this paper: the ECM deposition by the tumor cells and lysis. For the ECM deposition we take into account that the tumor cells consume more liquid to deposit their own matrix, hence the choice of a mass exchange from the liquid phase directly to the solid phase seems to be the easiest way: $\underset{E C M}{M}$. The lysis is the conversion of the necrotic tumor cells in liquid, hence the introduction of a mass exchange from the tumor phase to the liquid phase is required: $M_{l \rightarrow l} l$.

The new mass balance equation of the ECM is hence

$$
\frac{\partial \varepsilon}{\partial t}=\nabla \cdot \mathbf{v}^{\bar{s}}+\frac{(1-\varepsilon)}{\rho^{s}} \frac{\partial \rho^{s}}{\partial t}-\nabla \cdot\left(\varepsilon \mathbf{v}^{\bar{s}}\right)-\frac{\stackrel{l \rightarrow s}{M}}{E C M}
$$

The new mass balance equation of TCs reads 


$$
\begin{aligned}
& {\left[\frac{\varepsilon S^{t}}{K_{t}}+\frac{S^{t}(1-\varepsilon)}{K_{s}}\left(S^{t}+p^{t h} \frac{\partial S^{t}}{\partial p^{t h}}\right)+\varepsilon \frac{\partial S^{t}}{\partial p^{t h}}\right] \frac{\partial p^{t h}}{\partial t}} \\
& +\left[\frac{\varepsilon S^{t}}{K_{t}}+\frac{S^{t}(1-\varepsilon)}{K_{s}}\left(1-S^{l}-p^{h l} \frac{\partial S^{l}}{\partial p^{h l}}\right)\right] \frac{\partial p^{h l}}{\partial t} \\
& +\left[\frac{\varepsilon S^{t}}{K_{t}}+\frac{S^{t}(1-\varepsilon)}{K_{s}}\right] \frac{\partial p^{l}}{\partial t}=\nabla \cdot\left[\frac{k_{r e l}^{t} \mathbf{k}}{\mu^{t}} \cdot \nabla\left(p^{l}+p^{h l}+p^{t h}\right)\right] \\
& -S^{t}\left(\mathbf{1}: \mathbf{d}^{\overline{\bar{s}}}\right)-\nabla S^{t} \cdot\left(\varepsilon \mathbf{v}^{\bar{s}}\right)+\frac{1}{\rho^{t}}\left(\begin{array}{c}
l \rightarrow t \\
\stackrel{M}{M}-\stackrel{t \rightarrow l}{M} \\
\text { growth }
\end{array}\right)+S_{\text {lysis }}^{t} \frac{\stackrel{M}{E C M}}{\rho^{s}}
\end{aligned}
$$

The new mass balance equation of HCs is

$$
\begin{aligned}
& {\left[\frac{S^{h}(1-\varepsilon)}{K_{s}}\left(S^{t}+p^{t h} \frac{\partial S^{t}}{\partial p^{t h}}\right)-\varepsilon \frac{\partial S^{t}}{\partial p^{t h}}\right] \frac{\partial p^{t h}}{\partial S^{t}}} \\
& +\left[\frac{\varepsilon S^{h}}{K_{h}}+\frac{S^{h}(1-\varepsilon)}{K_{s}}\left(1-S^{l}-p^{h l} \frac{\partial S^{l}}{\partial p^{h l}}\right)-\varepsilon \frac{\partial S^{l}}{\partial p^{h l}}\right] \frac{\partial p^{h l}}{\partial S^{h}} \\
& +\left[\frac{\varepsilon S^{h}}{K_{h}}+\frac{S^{h}(1-\varepsilon)}{K_{s}}\right] \frac{\partial p^{l}}{\partial t}=\nabla \cdot\left(\frac{k_{r e l}^{h} \mathbf{k}}{\mu^{h}} \cdot \nabla\left(p^{l}+p^{h l}\right)\right) \\
& -S^{h}\left(\mathbf{1}: \mathbf{d}^{\overline{\bar{s}}}\right)-\nabla S^{h} \cdot\left(\varepsilon \mathbf{v}^{\bar{s}}\right)+S^{h} \frac{\stackrel{M}{M}}{\rho^{s}}
\end{aligned}
$$

The new mass balance equation of IF reads

$$
\begin{aligned}
& \frac{S^{l}(1-\varepsilon)}{K_{S}}\left(p^{t h} \frac{\partial S^{t}}{\partial p^{t h}}+S^{t}\right) \frac{\partial p^{t h}}{\partial t} \\
& +\left[\frac{S^{l}(1-\varepsilon)}{K_{S}}\left(1-p^{h l} \frac{\partial S^{l}}{\partial p^{h l}}-S^{l}\right)+\varepsilon \frac{\partial S^{l}}{\partial p^{h l}}\right] \frac{\partial p^{h l}}{\partial t} \\
& +\left(\frac{S^{l}(1-\varepsilon)}{K_{s}}+\frac{\varepsilon S^{l}}{K_{l}}\right) \frac{\partial p^{l}}{\partial t}=\nabla \cdot\left[\frac{k_{r e l}^{l} \mathbf{k}}{\mu^{l}} \cdot \nabla p^{l}\right] \\
& -S^{l}\left(\mathbf{1}: \mathbf{d}^{\overline{\bar{s}}}\right)-\nabla S^{l} \cdot\left(\varepsilon \mathbf{v}^{\bar{s}}\right)-\frac{1}{\rho^{l}}(\underset{\text { growth }}{\stackrel{l \rightarrow t}{M}}-\stackrel{t \rightarrow l}{\stackrel{M}{M}})-\frac{1}{\rho^{l}} \underset{E C M}{\stackrel{l \rightarrow s}{M}}
\end{aligned}
$$

where $\mu^{\alpha}$ is the dynamic viscosity, $k_{r e l}^{\alpha}$ is relative permeability, $\rho^{\alpha}$ is the density, $p^{\alpha}$ is the pressure of the $\alpha$ phase ( $\alpha=h, l$ and $t$ ) and $p^{\alpha \beta}$ is the differential pressure of phases $\alpha \beta$. Also, $\mathbf{d}^{\overline{\bar{s}}}$ is the Eulerian rate of strain tensor, $\mathbf{k}$ the intrinsic permeability tensor of the ECM, and $\underset{\text { growth }}{\stackrel{l \rightarrow t}{M}}$ is an inter-phase exchange of mass between the phases $l$ and $t$, and represents the mass of IF which becomes tumor due to cell growth. $K_{i}$ is the compressibility of phase $i$ (with $i=s, t, h$ and $l$ ). For all other terms refer to the abbreviations.

Summing Eqs. (4-6), using the constraint equations on volume fractions and saturation, Eqs. $(1,2)$ gives (see [22]) 


$$
\begin{aligned}
& {\left[\frac{\varepsilon S^{t}}{K_{t}}+\frac{1-\varepsilon}{K_{s}}\left(S^{t}+p^{t h} \frac{\partial S^{t}}{\partial p^{t h}}\right)\right] \frac{\partial p^{t h}}{\partial t}} \\
& +\left[\frac{\varepsilon S^{t}}{K_{t}}+\frac{\varepsilon S^{h}}{K_{h}}+\frac{1-\varepsilon}{K_{s}}\left(1-S^{l}-p^{h l} \frac{\partial S^{l}}{\partial p^{h l}}\right)\right] \frac{\partial p^{h l}}{\partial t} \\
& +\left(\frac{\varepsilon S^{t}}{K_{t}}+\frac{\varepsilon S^{h}}{K_{h}}+\frac{\varepsilon S^{l}}{K_{l}}+\frac{1-\varepsilon}{K_{s}}\right) \frac{\partial p^{l}}{\partial t}=\nabla \cdot\left[\frac{k_{r e l}^{t} \mathbf{k}}{\mu^{t}} \cdot \nabla p^{t h}\right] \\
& +\nabla \cdot\left[\left(\frac{k_{r e l}^{t} \mathbf{k}}{\mu^{t}}+\frac{k_{r e l}^{h} \mathbf{k}}{\mu^{h}}\right) \cdot \nabla p^{h l}\right]+\nabla \cdot\left[\left(\frac{k_{r e l}^{t} \mathbf{k}}{\mu^{t}}+\frac{k_{r e l}^{h} \mathbf{k}}{\mu^{h}}+\frac{k_{r e l}^{l} \mathbf{k}}{\mu^{l}}\right) \cdot \nabla p^{l}\right] \\
& -\left(\mathbf{1}: \mathbf{d}^{\overline{\bar{s}}}\right)+\frac{\rho^{l}-\rho^{t}}{\rho^{t} \rho^{l}}\left(\begin{array}{c}
\stackrel{l \rightarrow t}{M} \\
g \text { growth }
\end{array} \underset{\text { lysis }}{\stackrel{t \rightarrow l}{M}}\right)+\frac{\rho^{l}-\rho^{s}}{\rho^{s} \rho^{l}} \underset{E C M}{\stackrel{M}{M}}
\end{aligned}
$$

Equations (4, 5 and 7) which incorporate Eq. (3) are three of the governing equations of the model.

The tumor phase $t$ comprises a necrotic compartment (NTC) with mass fraction $\omega^{N \bar{t}}$ and a growing compartment with living cells (LTC) whose mass fraction is $1-\omega^{N \bar{t}}$. Assuming that there is no diffusion of either necrotic or living cells and that there is no exchange of the necrotic cells with other phases, the mass balance equations arefor the necrotic tumor cells:

$$
\frac{\partial\left(\varepsilon^{t} \rho^{t} \omega^{N \bar{t}}\right)}{\partial t}+\nabla \cdot\left(\varepsilon^{t} \rho^{t} \omega^{N \bar{t}} \mathbf{v}^{\bar{t}}\right)=\varepsilon^{t} r^{N t}-\stackrel{t \rightarrow l}{M}
$$

for the living tumor cells:

$$
\frac{\partial\left[\varepsilon^{t} \rho^{t}\left(1-\omega^{N \bar{t}}\right)\right]}{\partial t}+\nabla \cdot\left[\varepsilon^{t} \rho^{t}\left(1-\omega^{N \bar{t}}\right) \mathbf{v}^{\bar{t}}\right]=\underset{\text { growth }}{\stackrel{l \rightarrow t}{M}}-\varepsilon^{t} r^{N t}
$$

where $\mathbf{v}^{\bar{t}}$ is the velocity of the tumor cells (LTC and NTC move with the same velocity) and $\varepsilon^{t} r^{N t}$ is the death rate of tumor cells, i.e. the rate of generation of necrotic cells. The reaction term $\varepsilon^{t} r^{N t}$ is an intra-phase exchange term (see [22]).

The sum of the previous two equations gives the equation for tumor cells:

$$
\frac{\partial\left(\varepsilon S^{t} \rho^{t}\right)}{\partial t}+\nabla \cdot\left(\varepsilon S^{t} \rho^{t} \mathbf{v}^{\bar{t}}\right)=\underset{\text { growth }}{\stackrel{l \rightarrow t}{M}}-\stackrel{t \rightarrow l}{M} \text { lysis }
$$

From this equation, after the introduction of (3) and taking into account of the differential pressures as primary variables, see [22], we have obtained Eq. (4).

After the introduction of Eq. (10), Eq. (8) for the necrotic tumor cells can be rewritten:

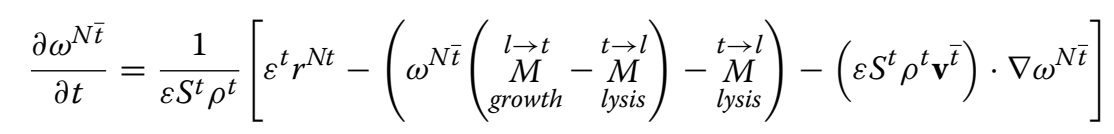

where $\underset{\text { lysis }}{\stackrel{t \rightarrow}{M}}$ takes into account of mass exchange between the necrotic compartment of tumor cells and the IF phase.

The mass balance equation of the nutrient reads 


$$
\begin{aligned}
& \varepsilon S^{l} \frac{\partial \omega^{n l}}{\partial t}-\nabla \cdot\left(\varepsilon S^{l} D_{e f f}^{n l} \nabla \omega^{n l}\right)
\end{aligned}
$$

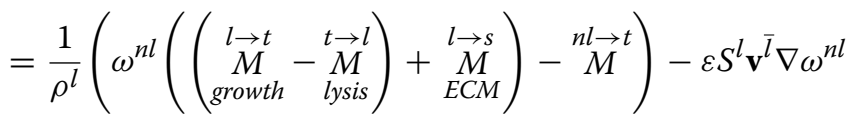

where $D_{e f f}^{\overline{n l}}$ is the effective diffusivity of the nutrient species in the extracellular space and $\stackrel{n l \rightarrow t}{M}$ is the mass of nutrient consumed by tumor cells via metabolism and growth. Eq. (12) is another governing equation of the model.

The effective stress tensor, $\mathbf{t}_{\text {eff }}^{\overline{\bar{s}}}$, in the sense of porous media mechanics is

$$
\mathbf{t}_{e f f}^{\overline{\bar{s}}}=\mathbf{t}^{\overline{\bar{s}}}+\bar{\alpha} p^{s} \mathbf{1}
$$

where $\mathbf{1}$ is the unit tensor, $\mathbf{t}^{\overline{\bar{s}}}$ is the total stress tensor in the solid phase, $\bar{\alpha}$ is the Biot's coefficient $\bar{\alpha}=1-K / K_{s}$, with $K$ the compressibility of the empty ECM. In the modeled problem, $K / K_{s}$ tends to zero hence we can assume a Biot's coefficient equal to 1 . The solid pressure $p^{s}$ is given as [26]

$$
p^{s}=S^{h} p^{h}+S^{t} p^{t}+S^{l} p^{l}=p^{l}+\left(1-S^{l}\right) p^{h l}+S^{t} p^{t h}
$$

where the Bishop parameter of each fluid phase (solid surface fraction in contact with the phase) has been taken equal to its own degree of saturation.

The last governing equation of the model is the linear momentum balance of the solid phase expressed in rate form [28] as

$$
\nabla \cdot\left(\frac{\partial \mathbf{t}_{e f f}^{\overline{\bar{s}}}}{\partial t}-\bar{\alpha} \frac{\partial p^{s}}{\partial t} \mathbf{1}\right)=0
$$

where the interaction between the solid and the three fluid phases is accounted for through the effective stress principle, Eq. (13).

\section{Solid phase behavior}

Since an ECM is present in the model its configuration is used as reference configuration whereby the velocities of the fluid are relative to the ECM which is described in a Lagrangian system. This is customary in multiphase flow models within deforming porous media [28].

A large deformation regime is assumed for the ECM (solid phase). As far as the stress and strain measures are concerned, objectivity (invariancy with respect to coordinate transformations, particularly rotations) and work-conjugacy (which guarantees energy consistency, i.e. correct expressions for the second-order energy increment) should be conserved. The satisfaction of the second requirement guarantees that of the first one, but not the opposite. Among the several objective stress rates [29, 30] we take the Truesdell stress rate.

It is recalled that the deformation is described by the velocity gradient tensor $\mathbf{L}^{\overline{\bar{s}}}=\nabla \mathbf{v}^{\overline{\bar{s}}}=\mathbf{d}^{\overline{\bar{s}}}+\mathbf{w}^{\overline{\bar{s}}}$, where the symmetric part $\mathbf{d}^{\overline{\bar{s}}}$ is the Eulerian strain rate tensor 


$$
d_{i j}^{s}=\frac{1}{2}\left(\frac{\partial v_{i}}{\partial x_{j}}+\frac{\partial v_{j}}{\partial x_{i}}\right)
$$

and the skew-symmetric one, $\mathbf{w}^{\overline{\bar{s}}}$, the spin tensor

$$
w_{i j}^{s}=\frac{1}{2}\left(\frac{\partial v_{i}}{\partial x_{j}}-\frac{\partial v_{j}}{\partial x_{i}}\right)
$$

The objective Truesdell rate of Cauchy stress is

$$
T \dot{\mathrm{t}}_{i j}^{s}=\dot{\mathrm{t}}_{i j}^{s}-\mathrm{t}_{k j}^{s} \frac{\partial v_{i}}{\partial x_{k}}-\mathrm{t}^{s} \frac{\partial v_{i}}{\partial x_{k}}+\mathrm{t}_{i j}^{s} \frac{\partial v_{k}}{\partial x_{k}}
$$

which is work conjugate with the Green-Lagrange strain measure. For a sufficiently small loading step (or increment), one may use the deformation rate tensor (or velocity strain) $\mathbf{d}^{\overline{\bar{s}}}$ or the increment representing the linearized strain increment from the initial (stressed and deformed) state in the step, i.e. the last converged solution

$$
\Delta E_{i j}^{s}=d_{i j}^{s} \Delta t
$$

The adoption of the objective Truesdell rate of Cauchy gives a symmetric tangent stiffness matrix.

\section{Constitutive relationships}

The constitutive relationships for the fluid phases have been extensively dealt with in $[17,18]$. The constitutive equations for growth, necrosis, and nutrient consumption are given in $[15,17]$ and only the new relationships are listed here.

As we have seen in section "General governing equations" we introduce a new term for the mass exchange between the tumor cell phase and the liquid phase to take into account of lysis. The constitutive relationship for this mass exchange is:

$$
\stackrel{t \rightarrow l}{\underset{\text { lysis }}{l}}=\lambda \omega^{N t} \varepsilon S^{t}
$$

with $\lambda$ constant, and $\omega^{N t} \varepsilon S^{t}$ the mass fraction of necrotic tumor cells. The mass exchange is proportional to the volume fraction of the necrotic cells present; hence a part of the necrotic cells becomes new liquid and give new sustenance to the living tumor cells.

The other new mass exchange term is that between liquid and solid phase to take into account of the ECM growth. This term is assumed as:

$$
\underset{E C M}{\stackrel{l \rightarrow s}{M}}=\gamma\left\langle\frac{\varepsilon_{0}^{s}-\varepsilon^{s}}{\varepsilon_{0}^{s}}\right\rangle \varepsilon S^{t}
$$

with $\varepsilon_{0}^{s}$ constant, being the ECM production limit and $\gamma$ the coefficient of ECM deposition. This form considers that the new ECM is produced by the tumor cells, hence the mass exchange is proportional to the TCs mass fraction with a factor $\gamma$. The function into the Macaulay brackets puts a limit for the ECM production; $\varepsilon_{0}^{s}$ is the superior limit; when the solid mass fraction reaches this limit the production of ECM stops. Below this value the production is inversely proportional to the solid mass fraction. 
The solid phase behavior is either Green-elastic where the elastic coefficients are derived from the strain energy function or elasto-visco-plastic where the stress-strain relation is defined incrementally. The absence of a potential in the second case requires the adoption of objective stress rates mentioned above. Elasto-visco-plasticity is appropriate for remodeling of the ECM, see Preziosi et al. [31]. The elasto-visco-plastic model has been described in [22] in detail hence only the principal points are summarized here together with the introduction of a new expression of the elastic modulus.

The Eulerian strain rate tensor defined in Eq. (16) is composed of an elastic and an irreversible part, see [32]. Hence in each step of our updated Lagrangian analysis with small strain increments, the total solid strain can be considered as a sum of the elastic part and the visco-plastic part.

$$
\mathbf{e}^{\overline{\bar{s}}}=\mathbf{e}_{e l}^{\overline{\bar{s}}}+\mathbf{e}_{v p}^{\overline{\bar{s}}}
$$

The relationship between the effective stress for the solid and the elastic strain is

$$
\dot{\mathbf{t}}_{e f f}^{\overline{\bar{s}}}=\mathbf{D}_{\mathrm{s}}: \dot{\mathbf{e}}_{e l}^{\overline{\bar{s}}}=\mathbf{D}_{\mathrm{s}}:\left(\dot{\mathbf{e}}^{\overline{\bar{s}}}-\dot{\mathbf{e}}_{v p}^{\bar{s}}\right)
$$

where $\mathbf{D}_{\mathrm{s}}$ is the tangent matrix containing the mechanical properties of the solid skeleton.

For visco-plastic analysis the constitutive tangent matrix $\mathbf{D}_{\mathrm{s}}$ should be such that all material symmetries are preserved, in accordance with the associative character of the model. The matrix will generally depend on the current state variables and on the direction of loading.

The elastic range is defined as the set of all possible absolute values of the effective stress that are less than or equal to the frictional constant $\mathrm{t}_{e f f, y^{\mathrm{s}}}^{\mathrm{s}}$ i.e. the yield limit (this value allows to define the boundary of elastic domain) [22]. Until the absolute value of the effective stress is contained in the elastic range, the rate of change of the visco-plastic strain is zero while beyond this limit it is different from zero.

In the elastic range the mechanical elastic behavior is governed by the Young modulus that is not constant but it is proportional to the mass fraction of the solid phase:

$$
E\left(\varepsilon^{s}\right)=\frac{E_{f}}{\varepsilon_{\text {fin }}^{s}}\left\langle\frac{\varepsilon^{s}-\varepsilon_{i}^{s}}{1-\varepsilon_{i}^{s}}\right\rangle
$$

In this equation $E_{f}$ is the final value of the Young modulus, $\varepsilon_{f i n}^{s}$ is the final mass fraction of solid and $\varepsilon_{i}^{s}$ is the mass fraction of solid below which the ECM has negligible mechanical properties. This function has been chosen with reference to the variable Young modulus in [33].

The particular elasto-visco-plastic model used to describe the ECM behavior was introduced by Perzyna $[34,35]$. Here it is used by introducing the von Mises yield condition as limit yield function, above which the ECM has viscoplastic behavior. The algorithm used to model this constitutive behavior is the "radial return mapping algorithm" by Simo and Hughes [35] and has been implemented in the finite elements code CAST3 M (http://www.cast3m.cea.fr) of the French Atomic Energy Commission together with our model. 
An overview of the finite element implementation and solution process is given in the "Appendix".

\section{Results and discussion}

This section shows numerical results of the model used to simulate the multicellular tumor spheroid (MTS) growing in vitro. At the beginning, a comparison with experimental data validates the model and then two cases of MTS growth are analyzed. In these examples the focus is on the ECM deposition and lysis effects.

\section{MTS growth in vitro: comparison with experimental data}

The model had been validated with respect to the experiment by Chignola et al. 2000 [21] in [22]. With the present version of the model we reproduce the new available data of MTS growth experiments in vitro carried out at HMRI. Differently from [15, 22] the simulation is performed with free-boundary conditions; this means that we simulate only the MTS, without IF or ECM outside the border, see Fig. 2. The rim of MTS moves during the time with velocity:

$$
v_{t}=-\frac{k_{r e l}^{t} \mathbf{k}}{\mu^{t} \varepsilon_{t}} \cdot \nabla p^{t h}
$$

Hence the boundary conditions are updated at every step and have fixed values on the moving boundary: zero IF pressure and oxygen concentration equal to $7.0 \times 10^{-6} \mathrm{~Pa}$.

The initial conditions in the MTS zone are: volume fraction of TCs equal to 0.01, volume fraction of $\mathrm{HC}$ equal to 0 , porosity equal to 1 . The parameters for this simulation are

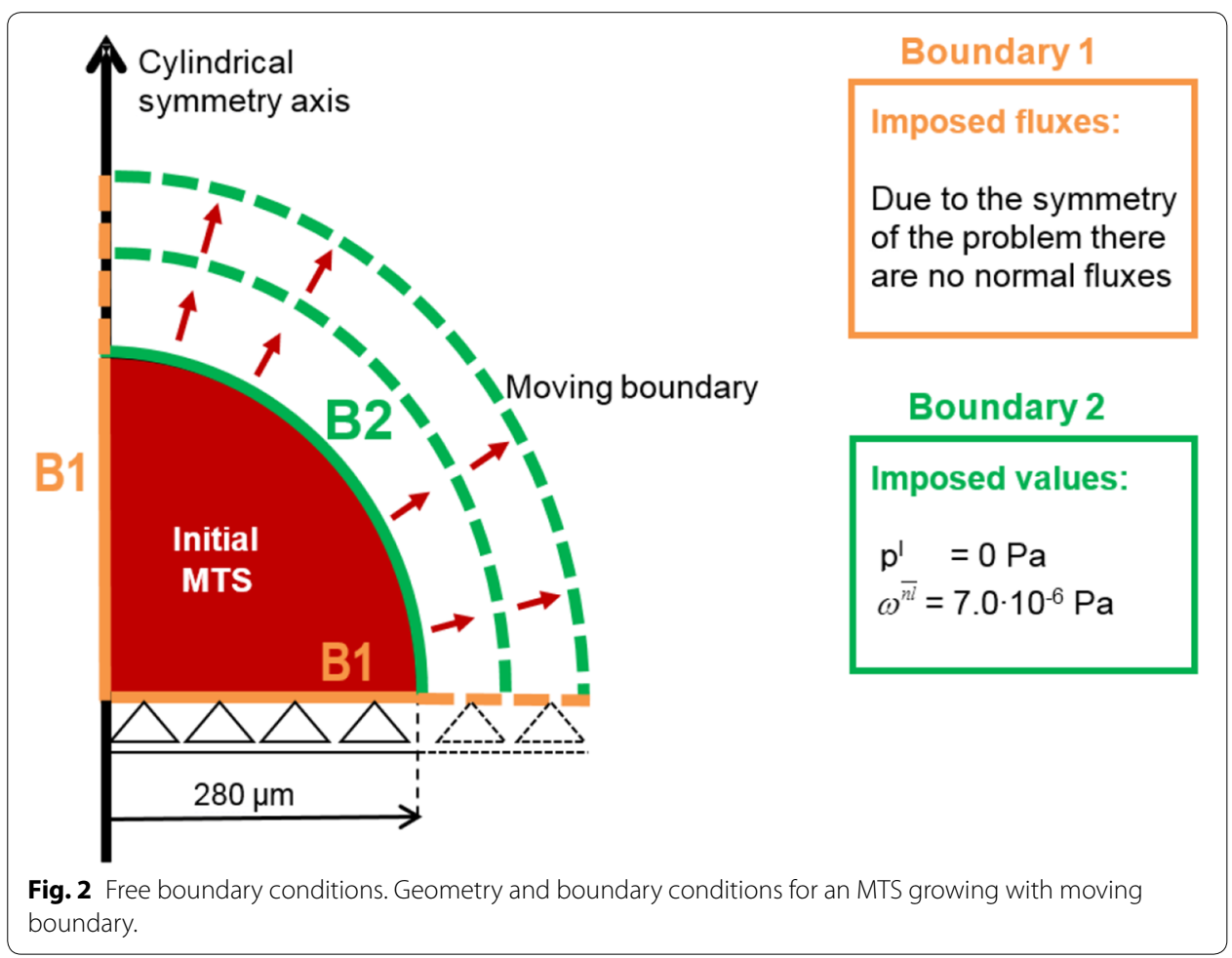


reported in Table 1 . The parameters for TCs, HCs, IF and oxygen are listed in Tables 2, 3. In this case with free boundary conditions $\lambda$ affects only marginally the behavior as has been evidenced with two values of $\lambda=0.1$ and $\lambda=0.001$.

A deformable ECM is assumed and it is deposed by TCs during the tumor mass growth according to Eq (21). For the ECM the parameters are presented in Table 4. The Young's modulus is of the same order of magnitude of the corresponding one in [22].

The results are shown in Fig. 3 where dots refer to the diameter of U87 spheroid. Human multiforme glioblastoma U-87 MG cells (ATCC) were grown at $37^{\circ} \mathrm{C}$ at $5 \%$ CO2 in complete EMEM (HyClone) supplemented with $50 \mathrm{U} / \mathrm{mL}$ penicillin, $50 \mu \mathrm{g} / \mathrm{mL}$ streptomycin and 10\% FBS (v/v). Multicellular U-87 MG spheroids prepared by liquid overlay method. Briefly, serum free EMEM medium with $2 \%(\mathrm{w} / \mathrm{v})$ agar was prepared and sterilized. This agar solution was added to the bottom of each well of the 24 wellplates to prevent cell adhesion onto the well surface. Plates were allowed to cool down before use. U-87 MG cells were counted and plated at the density of 20,000 cells/well in

Table 1 Initial conditions

\begin{tabular}{lllll}
\hline Zone & $\boldsymbol{p}^{l}[\mathrm{~Pa}]$ & $\boldsymbol{S}^{h}[-]$ & $\boldsymbol{S}^{t}[-]$ & $\boldsymbol{\omega}^{\overline{n^{\prime}}}[-]$ \\
\hline Red zone (up to 280 $\mu \mathrm{m})$ & 0.0 & 0.0 & 0.01 & $7.0 \times 10^{-6}$ \\
\hline
\end{tabular}

Table 2 Parameters depending on cells' type and IF

\begin{tabular}{|c|c|c|c|}
\hline Parameter & Symbol & Value & Unit \\
\hline Density of the three fluid phases $(\alpha=h, t$ and $l)$ & $\rho^{\alpha}$ & 1,000 & $\mathrm{~kg} / \mathrm{m}^{3}$ \\
\hline Dynamic viscosity of IF [22] & $\mu^{\prime}$ & $1 \times 10^{-3}$ & Pa s \\
\hline Dynamic viscosity of TC [22] & $\mu_{0}^{t}$ & 36 & Pa s \\
\hline Dynamic viscosity of HC [22] & $\mu_{0}^{h}$ & 36 & Pas \\
\hline Adhesion of TC [22] & $\psi^{t}$ & 0 & $\mathrm{~Pa} / \mathrm{m}$ \\
\hline Adhesion of HC [22] & $\psi^{h}$ & 0 & $\mathrm{~Pa} / \mathrm{m}$ \\
\hline Critical mass fraction of oxygen $[15,17]$ & $\omega_{\text {crit }}^{\overline{n l}}$ & $6.0 \times 10^{-6}$ & - \\
\hline Growth coefficient of tumor cells $[15,17]$ & $\gamma_{\text {growth }}^{t}$ & $1.3 \times 10^{-2}$ & $\mathrm{~kg} /\left(\mathrm{m}^{3} \mathrm{~s}\right)$ \\
\hline Necrosis coefficient $[15,17]$ & $\gamma_{\text {necrosis }}^{t}$ & $1.0 \times 10^{-2}$ & $\mathrm{~kg} /\left(\mathrm{m}^{3} \mathrm{~s}\right)$ \\
\hline Consumption related to growth $[15,17]$ & $\gamma_{\text {growth }}^{\overline{n l}}$ & $5 \times 10^{-4}$ & $\mathrm{~kg} /\left(\mathrm{m}^{3} \mathrm{~s}\right)$ \\
\hline Consumption related to metabolism $[15,17]$ & $\gamma_{0}^{\overline{n l}}$ & $6 \times 10^{-5}$ & $\mathrm{~kg} /\left(\mathrm{m}^{3} \mathrm{~s}\right)$ \\
\hline HC-IF interfacial tension [22] & $\sigma_{h l}$ & 72 & $\mathrm{mN} / \mathrm{m}$ \\
\hline TC-HC interfacial tension [22] & $\sigma_{t h}$ & 36 & $\mathrm{mN} / \mathrm{m}$ \\
\hline TC-IF interfacial tension & $\sigma_{t /}$ & 108 & $\mathrm{mN} / \mathrm{m}$ \\
\hline Lysis parameter & $\lambda$ & 0.001 & - \\
\hline
\end{tabular}

Table 3 Parameters related to oxygen diffusion

\begin{tabular}{llll}
\hline Parameter & Symbol & Value & Unit \\
\hline Diffusion coefficient of oxygen in interstitial fluid $[15,17]$ & $D_{0}^{\overline{n l}}$ & $3.2 \times 10^{-9}$ & $\mathrm{~m}^{2} / \mathrm{s}$ \\
Coefficient $\delta[15,17]$ & $\delta$ & 2 & - \\
Normal mass fraction of oxygen in tissue $[15,17]$ & $\omega_{\text {env }}^{\bar{n}}$ & $7.0 \times 10^{-6}$ & - \\
\hline
\end{tabular}


Table 4 Parameters for ECM taken from [22]

\begin{tabular}{llll}
\hline Parameter & Symbol & Value & Unit \\
\hline Density of the solid phase & $\rho^{s}$ & $1 \times 10^{3}$ & $\mathrm{~kg} / \mathrm{m}^{3}$ \\
Poisson's ratio of the ECM & $v$ & 0.4 & - \\
Young's modulus of the ECM & Efin & $1.6 \times 10^{2}$ & $\mathrm{~Pa}$ \\
Volume fraction of ECM (initial) & $\varepsilon^{5}$ & 0.0 & - \\
Coefficient $a$ & $a$ & 590 & $\mathrm{~Pa}$ \\
Intrinsic permeability & $k$ & $1.8 \times 10^{-15}$ & $\mathrm{~m}{ }^{2}$ \\
Yield effective stress limit & $\mathrm{t}_{\text {eff'y }}^{5}$ & $0.5 \times 10^{1}$ & $\mathrm{~Pa}$ \\
Viscosity & $\eta$ & 5 & $\mathrm{~Pa} \mathrm{~s}$ \\
Hardening modulus & $H$ & $1.0 \times 10^{2}$ & $\mathrm{~Pa}$ \\
\hline
\end{tabular}

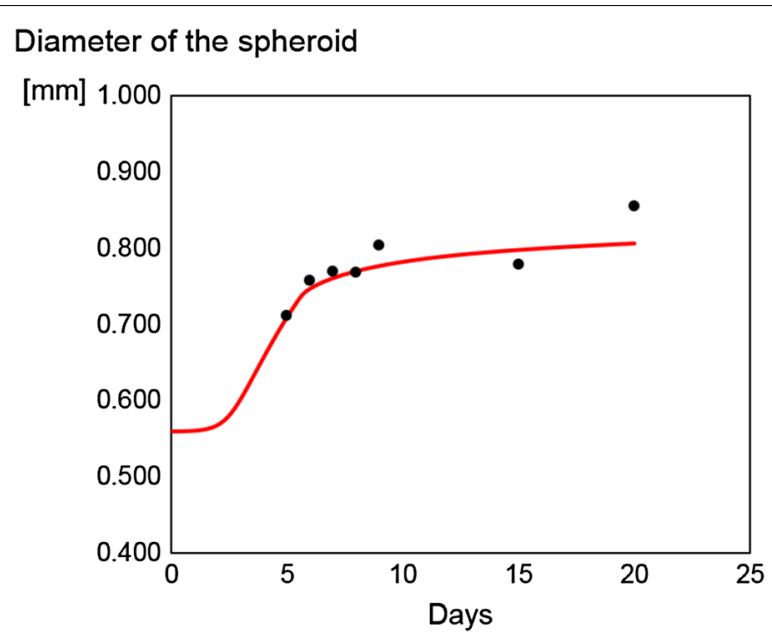

Fig. 3 Model results and experimental data. Comparison between model results (solid line) and experimental data (dots) U87 spheroid cultivated by C. Stigliano at HMRI.

complete EMEM. Plates were centrifuged for $5 \mathrm{~min}$ at $1,000 \times g$ to facilitate cell aggregation. Spheroid diameter was measured 5 days after cell seeding and over 20 days, using Nikon Eclipse Ti microscope (Nikon) with NIS-Element software. The culture medium was replaced with fresh complete medium every 3 days.

The simulation captures both the first exponential phase of the growth and the second phase: the plateau where the tumor mass reaches an equilibrium. The equilibrium, in the simulation, is reached thanks to the free-boundary conditions and to the growth limiter due to pressure. Lysis has here only a minor influence, contrarily to the last example. The moving boundary with a Dirichlet condition for the nutrient concentration at the MTS surface allows simulating growth without such a conditions posed at some point in the culture medium that may influence the proliferation and the movement of tumor cells.

A steady radius, as documented in several experiments (see for example [36-38]), characterizes the final phase of spheroid growth. This behavior is described by different models in the literature [39-42]. In [39] Byrne and Preziosi develop a biphasic model of avascular growth based on mixture theory. They report a steady radius for long times which depends on tumor compression and different model parameters. Another 
example is given by the work of Wise et al. [42], where a porous media model for tumor growth is derived by energetic considerations. Their solution displays the equilibrium radius as a function of cell adhesiveness and other parameters describing the growth of the tumor. In our previous work [22], we validated the model against experimental data describing the first stages of spheroid growth without considering the final phase. In this paper we extend the previous validation and take into account the complete growth pattern.

\section{Comparison between MTS growth in an ECM deposited by TCs, in an ECM-free medium and in an ECM scaffold}

In this example we compare the growth of (a) a multicellular tumor spheroid immersed in the interstitial fluid and deposing its own deformable ECM, (b) a multicellular tumor spheroid immersed in the interstitial fluid without ECM and (c) a multicellular tumor spheroid growing in a deformable and decellularized ECM scaffold. Tumor cells can in fact be grown successfully in a decellularized ECM of an organ as shown in Mishra et al. [43]. This allows mimicking the in vivo environment and has been done successfully for an ex vivo 3D lung model where it was possible to grow perfusable lung nodules [43].

The simulations are limited to the avascular stage. In this comparison we show the influence of the ECM presence on the growth.

The geometry of the three problems compared is simulated with a sphere segment in axisymmetric conditions with radius of $1,000 \mu \mathrm{m}$. At the initial time instant, in cases (a) and (b) the MTS is composed of only two phases: (1) the TC phase, in the red area with radius of $30 \mu \mathrm{m}$ shown in Fig. 4 , and (2) the IF phase which fills the whole domain; in case (c) the MTS is composed of the previous two phases and a third phase, (3) the solid phase ECM (which fills the whole domain). The atmospheric pressure is taken as the reference pressure and the initial IF pressure is zero $\mathrm{Pa}$ in the entire domain. The initial volume fraction of TCs is 0.02 , the HCs are not present and in cases (a) and (b) the porosity is 1 in the whole domain, while in case (c) the volume fraction of the ECM in the domain is 0.2 , hence the initial porosity is 0.8 .

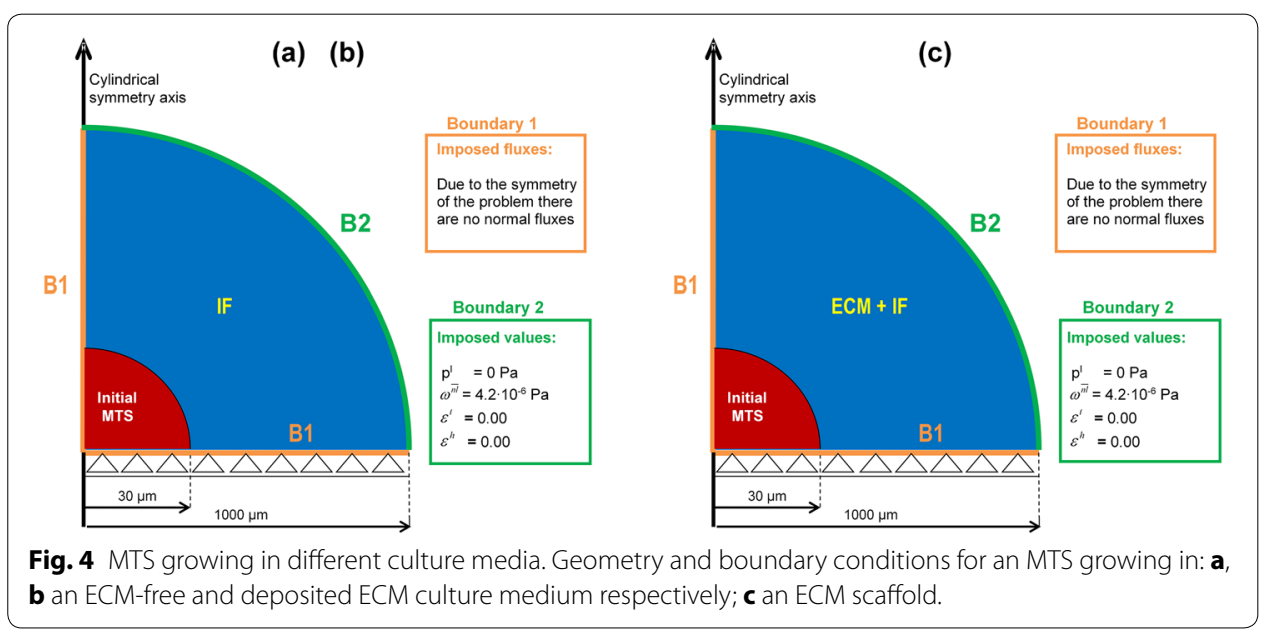


Boundary conditions are imposed as indicated in Fig. 4. To allow IF flux at the outer boundary the IF pressure is fixed there to zero Pa. Due to the symmetry of the problem there is no flux normal to the radius of the sphere segment. Oxygen is the sole nutrient species and its mass fraction is fixed to $\omega_{e n v}^{\overline{n l}}=4.2 \times 10^{-6}$ at the outer boundary and throughout the computational domain at initial time. This mass fraction of oxygen corresponds to the average of the dissolved oxygen in the plasma of a healthy individual.

All model parameters are listed in Tables 5, 6, 7 and 8, classified by type. The critical value of the oxygen mass fraction which controls TC growth rate and induces necrosis is $\omega_{c r i t}^{\overline{n l}}=3 \times 10^{-6}$ (the used constitutive equations for growth and necrosis are reported in [15]). This critical threshold has been chosen according with experiments of Walenta et al. [44]. Coefficients $\gamma_{\text {growth }}^{\overline{n l}}$ and $\gamma_{0}^{\overline{n l}}$ control the TCs uptake of oxygen (see the constitutive equation reported [16]); they allow modeling consumption due to TCs growth and their metabolism, and with the chosen values an oxygen consumption rate in accordance

Table 5 Initial conditions

\begin{tabular}{lllll}
\hline Zone & $\boldsymbol{p}^{\boldsymbol{l}}[\mathrm{Pa}]$ & $\boldsymbol{S}^{\boldsymbol{h}}[-]$ & $\boldsymbol{S}^{\boldsymbol{t}}[-]$ & $\boldsymbol{\omega}^{\overline{n^{\prime}}}[-]$ \\
\hline Red zone $($ up to $30 \mu \mathrm{m})$ & 0.0 & 0.0 & 0.02 & $4.2 \times 10^{-6}$ \\
Blue zone (up to 1,000 $\mu \mathrm{m})$ & 0.0 & 0.0 & 0.0 & $4.2 \times 10^{-6}$ \\
\hline
\end{tabular}

Table 6 Parameters depending on cells' type and IF

\begin{tabular}{llll}
\hline Parameter & Symbol & Value & Unit \\
\hline Density of the three fluid phases $(\alpha=h, t$ and $)$ & $\rho^{\alpha}$ & 1,000 & $\mathrm{~kg} / \mathrm{m}^{3}$ \\
Dynamic viscosity of IF [22] & $\mu^{\prime}$ & $1 \times 10^{-3}$ & $\mathrm{~Pa} \cdot \mathrm{s}$ \\
Dynamic viscosity of TC [22] & $\mu_{0}^{t}$ & 36 & $\mathrm{~Pa} \cdot \mathrm{s}$ \\
Dynamic viscosity of HC [22] & $\mu_{0}^{h}$ & 36 & $\mathrm{~Pa} \cdot \mathrm{s}$ \\
Adhesion of TC [22] & $\psi^{t}$ & 0 & $\mathrm{~Pa} / \mathrm{m}$ \\
Adhesion of HC [22] & $\psi^{h}$ & 0 & $\mathrm{~Pa} / \mathrm{m}$ \\
Critical mass fraction of oxygen [15, 17] & $\omega_{\text {crit }}^{\bar{n}}$ & $3.0 \times 10^{-6}$ & - \\
Growth coefficient of tumor cells [15, 17] & $\gamma_{\text {growth }}^{t}$ & $1.8 \times 10^{-2}$ & $\mathrm{~kg} /\left(\mathrm{m}^{3} \mathrm{~s}\right)$ \\
Necrosis coefficient [15, 17] & $\gamma_{\text {necrosis }}^{t}$ & $9.6 \times 10^{-3}$ & $\mathrm{~kg} /\left(\mathrm{m}^{3} \mathrm{~s}\right)$ \\
Consumption related to growth [15, 17] & $\gamma_{\text {growth }}^{\text {nl }}$ & $2 \times 10^{-4}$ & $\mathrm{~kg} /\left(\mathrm{m}^{3} \mathrm{~s}\right)$ \\
Consumption related to metabolism [15, 17] & $\gamma_{0}^{\bar{n}}$ & $3 \times 10^{-4}$ & $\mathrm{~kg} /\left(\mathrm{m}^{3} \mathrm{~s}\right)$ \\
HC-IF interfacial tension [22] & $\sigma_{\text {hl }}$ & 72 & $\mathrm{mN} / \mathrm{m}$ \\
TC-HC interfacial tension [22] & $\sigma_{\text {th }}$ & 36 & $\mathrm{mN} / \mathrm{m}$ \\
TC-IF interfacial tension & $\sigma_{t /}$ & 108 & $\mathrm{mN} / \mathrm{m}$ \\
Lysis parameter & $\lambda$ & 0.0001 & - \\
\hline
\end{tabular}

Table 7 Parameters related to oxygen diffusion

\begin{tabular}{llll}
\hline Parameter & Symbol & Value & Unit \\
\hline Diffusion coefficient of oxygen in interstitial fluid $[15,17]$ & $D_{0}^{\bar{n}}$ & $3.2 \times 10^{-9}$ & $\mathrm{~m}^{2} / \mathrm{s}$ \\
Coefficient $\delta[15,17]$ & $\delta$ & 2 & - \\
Normal mass fraction of oxygen in tissue $[15,17]$ & $\omega_{\text {env }}^{\overline{n l}}$ & $4.2 \times 10^{-6}$ & - \\
\hline
\end{tabular}


Table 8 Parameters for ECM taken from [22]

\begin{tabular}{llll}
\hline Parameter & Symbol & Value & Unit \\
\hline Density of the solid phase & $\rho^{s}$ & $1 \times 10^{3}$ & $\mathrm{~kg} / \mathrm{m}^{3}$ \\
Poisson's ratio of the ECM & $v$ & 0.4 & - \\
Young's modulus of the ECM in case of Fig. 4a & Efin & $1 \times 10^{2}$ & $\mathrm{~Pa}$ \\
Young's modulus of the ECM in case of Fig. 4b & $E$ & $1 \times 10^{2}$ & $\mathrm{~Pa}$ \\
Volume fraction of ECM (initial) in case of Fig. 4a & $\varepsilon^{5}$ & 0.0 & - \\
Volume fraction of ECM (initial) in case of Fig. 4b & $\varepsilon^{5}$ & 0.2 & - \\
Coefficient a [22] & $a$ & 590 & $\mathrm{~Pa}$ \\
Intrinsic permeability & $k$ & $1.8 \times 10^{-15}$ & $\mathrm{~m}^{2}$ \\
Yield effective stress limit & $\mathrm{t}_{\text {effly }}^{5}$ & $0.5 \times 10^{1}$ & $\mathrm{~Pa}$ \\
Viscosity & $\eta$ & 5 & $\mathrm{~Pa} \mathrm{~s}$ \\
Hardening modulus & $\mathrm{H}$ & $1.0 \times 10^{2}$ & $\mathrm{~Pa}$ \\
\hline
\end{tabular}

with experimental observations of Mueller-Klieser et al. [45] is obtained. The oxygen diffusion coefficient in the IF, $D_{0}^{\overline{n l}}$, has been taken from literature [46].

Recently we have also included the effect of fluid-fluid interfacial tension taking into account explicitly HC-IF, $\sigma_{h l}$, TC-HC, $\sigma_{t h}$, and TC-IF interfacial tensions, $\sigma_{t l}$ [17]. The values of interfacial tensions in Table 6 have been chosen respecting order of magnitude of experimental measurements $[47,48]$.

From Fig. 5a, by comparing the tumor mass fractions versus radius in case of ECM deposited by TCs (solid lines), in case of ECM-free culture medium (dotted lines) and in case of remodeling ECM scaffold (dashed lines), it appears that the MTS growth seems to be larger in the first case. This can be explained with the larger availability of IF to

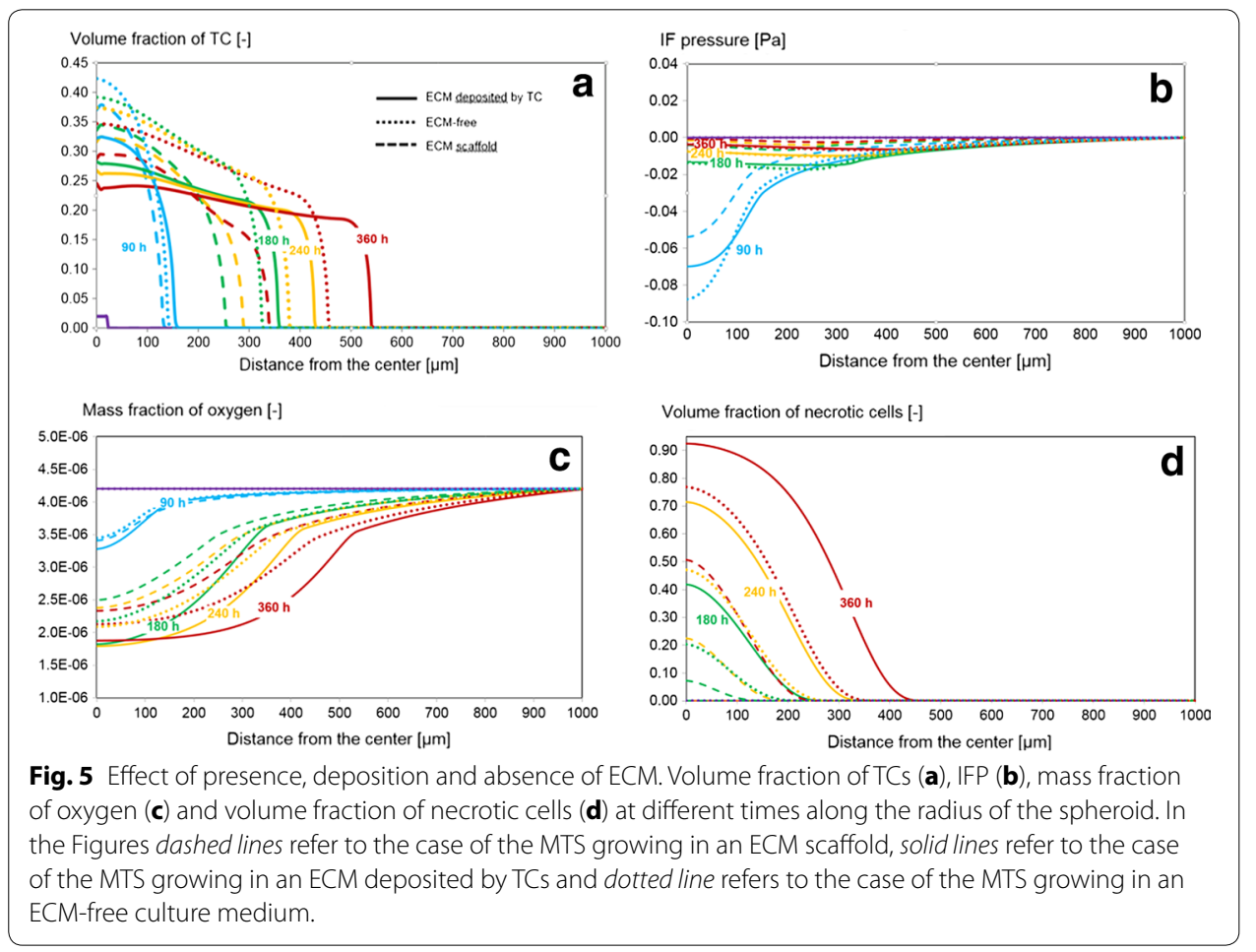


MTS growth in a deposited ECM and in a ECM-free medium, where the space outside TCs is filled only by IF; in a remodeling ECM scaffold there is also this scaffold. Furthermore, in the first two cases, the MTS has growth freedom; it must not deform an external ECM. The difference between the tumor growth in case of deposited ECM and in case of ECM-free medium may be explained as follows: the deposited ECM hinders the increase of TCs density in the center of MTS; the growing tumor cells are forced to the outside. Without ECM, instead, the tumor cells growth increases the malignant mass density more than its volume. Figure $5 b$ shows the IF pressure versus radius for the case of deposited ECM culture medium (solid lines), ECM-free culture medium (dotted lines) and the case of remodeling ECM scaffold (dashed lines). In all cases at the beginning IFP gradient remains close to zero because little additional IF from the surroundings is needed and the TCs increase density without lateral expansion. When the MTS increases its volume the IF flows inward because of increased consumption, respecting the constraint $S^{t}+S^{l}=1$. The processes in a ECM-free medium and in a deposited ECM are more rapid than in a remodeling ECM scaffold: the front of IF pressure and of tumor mass fraction move faster towards the outside border (see Fig. 5b).

Figure 5c shows the evolution of the oxygen mass fraction in a culture medium with deposited ECM, ECM-free culture medium and remodeling ECM scaffold (solid, dotted and dashed lines respectively). The oxygen decreases from the original mass fraction of $4.2 \times 10^{-6}$ because of its consumption made by living tumor cells. Oxygen is the sole nutrient species considered here. Once the oxygen concentration decreases below the critical value fixed in Table 6 cell necrosis begins. In Fig. $5 \mathrm{~d}$ we can see a larger necrosis in the MTS growing in deposited ECM and ECM-free culture medium (solid and dotted lines) with respect to the MTS growing in remodeling ECM scaffold. Indeed, TCs grow faster and consume more in the first two cases.

It is recalled that finite displacements and a Lagrangian updated formulation together with the objective Truesdell rate of Cauchy stress are adopted for the simulations, see section "Solid phase behavior". The Young modulus for the deformable deposited ECM during the MTS growth is described in Eq. (24) with Efin $=8.0 \times 10^{1} \mathrm{~Pa} E=8.0 \times 10^{1}$ Pa for the deformable ECM scaffold. Hence when the deposited ECM reaches the volume fraction of $\varepsilon_{f i n}^{s}=0.2$ the Young modulus is the same as that of the ECM scaffold with $\varepsilon^{s}=0.2$. The constitutive behavior of the deformable ECM is elastic until the yield limit, after that the behavior becomes viscoplastic. The viscoplastic parameters for the Perzyna type model (see [22]) are described in Table 8.

Figure 6 shows the evolution of the solid volume fraction; on the top for the case of ECM deposited by TCs and at the bottom for the case of remodeling ECM scaffold. In the first case the initial solid volume fraction is 0 because there is no solid phase. At the final stage the solid volume fraction is different from 0 in the zone where the TCs have grown and have deposited their ECM. The average solid volume fraction in this zone is 0.2 , because of the limit we have posed to the deposition, but we can see a larger solid volume fraction in the center with respect to the border of MTS: the limit of 0.2 is overcome by deformation. The TCs indeed have larger density in the center and hence the ECM has a larger density in the center too. In the second case the initial and final average solid volume fraction is fixed at 0.2 because the ECM scaffold is always present. At the final stage we observe however a smaller solid volume fraction in the center of the 


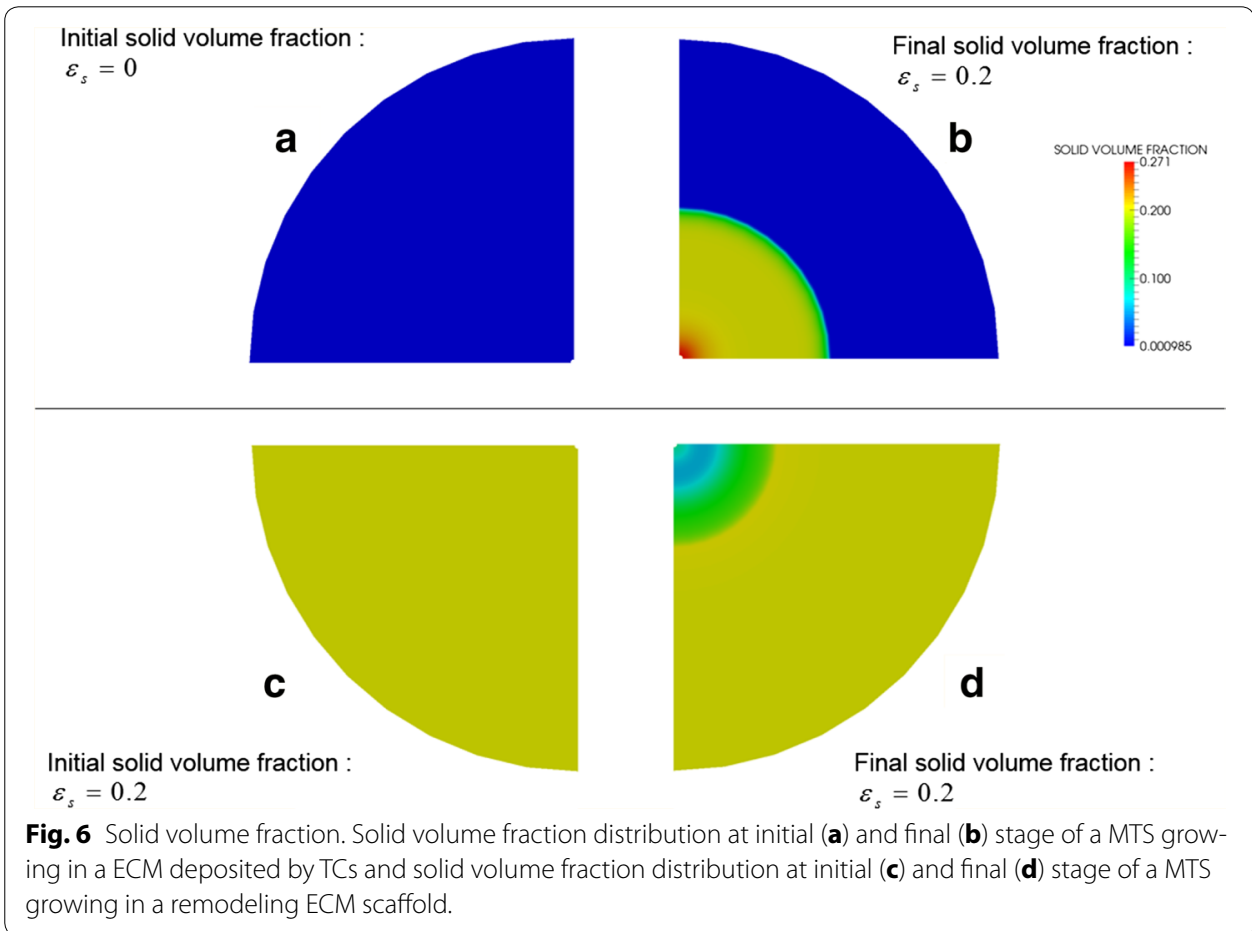

spheroid and larger solid volume fraction in the border due again to the deformability of the solid phase. In a deformable ECM the growing tumor is able to increase the available pore space in the region where growth happens, while in the outer space the porosity of the ECM slightly decreases (see [22]).

\section{Comparison between MTS growth in a deposited ECM medium with and without lysis}

In this section the focus is on the effect of the lysis in the MTS growth in a ECM deposited by TCs. We recall that lysis transforms a part of necrotic tumor cells in the core of MTS into liquid phase increasing the liquid pressure in the center of MTS; remind that with growing tumor density the permeability reduces hindering the outflow through the viable rim, see the constitutive law for permeability in [15]. Since the IF pressure is higher inside the tumor than at its outer boundary, the IF flows towards the boundary while tumor cells move towards the center of the MTS as required by the mass balance. The MTS growth reaches an equilibrium phase in the tumor cell flux between the center and the border of the MTS.

The data are the same as in the previous example, only the lysis parameter changes. The comparison is between the lysis parameter $\lambda$ of the Eq. (20), equal to 0.0001 and 0.01 . In the Fig. 7a the radius of the MTS versus the time is shown in both cases. An initial exponential growth followed by a linear phase can be seen. The plateau is not reached here because we did not use the free boundary condition, Eq. (25) which would hide partially the effect of lysis. In the case of lysis parameter $\lambda$ equal to 0.0001 the tumor grows more than in the case of the lysis parameter $\lambda$ equal to 0.01 : lysis transforms a part of necrotic cells in liquid phase and hence reduces the volume, and consequently the radius, of MTS. 


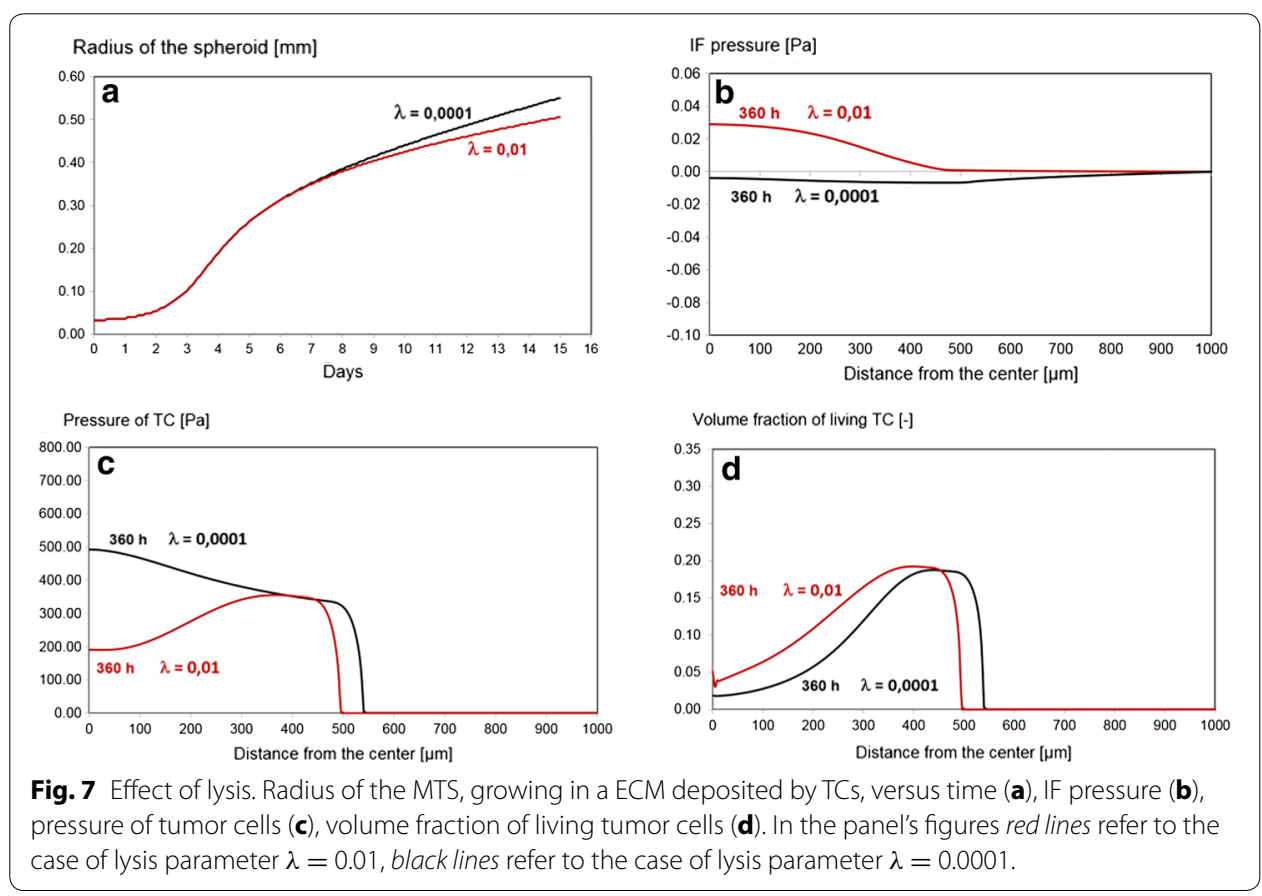

Lysis increases the liquid in the MTS, causing an increase of the IF pressure as shown in Fig. 7b. The red line represents the IF pressure at the last step of the analysis in the case of $\lambda$ equal to 0.01 ; the black line represents the IF pressure at last step in the case of $\lambda$ equal to 0.0001 . The increment of IF pressure happens in the necrotic core of MTS, where a part of necrotic cells become liquid and the outflow through the outer rim is reduced because of reduced permeability in the viable rim. The IF pressure passes from negative values of the black curve to positive ones in the red curve in the center of MTS. The IF pressures at the center of MTS, in both black and red curves are higher than at the border of the MTS; hence the IF flows from the center to the border. With the lysis parameter $\lambda=0.01$, this phenomenon is more visible.

In conclusion lysis produces outflow of the IF from the tumor mass. The tumor cells pressure however has an inverse behavior due to lysis: it decreases in the necrotic core as shown in Fig. 7c. Hence it redirects some of the tumor cells towards the interior. Figure $7 \mathrm{~d}$ shows the volume fraction of living tumor cells at the last calculation step: with $\lambda$ equal to 0.01 more tumor cells come back to the center of the MTS than with $\lambda$ equal to 0.0001 . The tumor cell flux resulting from proliferation has been conjectured also in [49].

\section{Conclusions}

The original model for tumor growth has been enhanced by the introduction of ECM deposition during the $\mathrm{TC}$ growth and of lysis, i.e. the re-transformation of necrotic cells into IF.

The ECM has been modeled as a porous solid matrix with Green-elastic and elastovisco-plastic material behavior within a large strain approach. Truesdell objective stress measure is adopted together with the deformation rate tensor. An updated Lagrangian formulation has been used for the numerical simulations. A free-boundary simulation 
of MTS growth with the enhanced mathematical formulation of the model has allowed to reproduce new experimental data, carried out at HMRI. Both the first exponential growth and the plateau when the tumor mass reaches equilibrium have been replicated.

ECM deposition, ECM free growth and ECM remodeling have then been investigated: it appears that the MTS growth seems to be faster in the first two cases. The larger availability of IF to MTS growth and the major growth freedom in a ECM-free medium with respect in a remodeling ECM medium can explain this behavior. The last example shows how lysis affects the results. Lysis transforms a part of the necrotic tumor cells into IF. This increases the liquid in the center of MTS, causing an increase of the IF pressure with respect to the border of the MTS. Hence the IF flows out from the center to the border and produces an outflow of the IF from the tumor mass. The tumor cell pressure instead results lower in the center of MTS because of lysis and this leads some of the tumor cells migrating towards the interior.

The introduction of lysis and ECM deposition allows capturing different aspects of the avascular tumor growth not yet comprised in the original model. We are now extending this more complete multiphase model to include the vascular stage of tumor growth.

\section{Nomenclature}

eqn. equation

eqs. equations

REV representative elementary volume

TCAT thermodynamically constrained averaging theory

a coefficient in the pressure-saturations relationship

$\mathrm{C}_{i j} \quad$ non linear coefficient of the discretized capacity matrix

$\mathbf{d}^{\overline{\bar{\alpha}}} \quad$ rate of strain tensor

$D_{e f f}^{\overline{i l}} \quad$ effective diffusion coefficient for the species i dissolved in the phase 1

$\mathbf{D}_{s} \quad$ tangent matrix of the solid skeleton

$\mathbf{e}^{\overline{\bar{s}}} \quad$ total strain tensor

$\mathbf{e}_{e l}^{\overline{\bar{s}}} \quad$ elastic strain tensor

$\mathbf{e}_{v p}^{\overline{\bar{s}}} \quad$ visco-plastic strain tensor

$\mathbf{f}_{v} \quad$ discretized source term associated to the primary variable $v$

$\mathbf{K}_{i j} \quad$ non linear coefficient of the discretized conduction matrix

$K_{i} \quad$ compressibility of the phase $i(i=s, t, h$ and $l)$

k intrinsic permeability tensor of the ECM

$k_{r e l}^{\alpha} \quad$ relative permeability of the phase $\alpha$

$\mathbf{N}_{v} \quad$ vector of shape functions related to the primary variable $v$

$p^{\alpha} \quad$ pressure in the phase $\alpha$

$p^{i j} \quad$ pressure difference between fluid phases $i$ and $j$

$\mathbf{R}^{\alpha} \quad$ resistance tensor

$S^{\alpha} \quad$ saturation degree of the phase $\alpha$

$\overline{\mathbf{t}}_{\text {eff }}^{\overline{\bar{s}}} \quad$ effective stress tensor of the solid phase $s$

$\mathbf{t}^{\overline{\bar{s}}} \quad$ total stress tensor of the solid phase $s$

$\mathbf{t}_{\text {eff }, y}^{\overline{\bar{s}}} \quad$ yield limit of the solid phase which defines the boundary of elastic domain

$\mathbf{u}^{s} \quad$ displacement vector of the solid phase $s$

$\mathbf{v}^{\bar{\alpha}} \quad$ velocity vector of the phase $\alpha$

$\mathbf{x} \quad$ solution vector

$\bar{\alpha} \quad$ Biot's coefficient 


\begin{tabular}{|c|c|}
\hline$\gamma_{\text {growth }}^{t}$ & growth coefficient \\
\hline$\gamma_{\text {necrosis }}^{t}$ & necrosis coefficient \\
\hline$\gamma_{\text {growth }}^{\overline{n l}}$ & nutrient consumption coefficient related to growth \\
\hline$\gamma_{0}^{\overline{n l}}$ & nutrient consumption coefficient not related to growth \\
\hline$\varepsilon$ & porosity \\
\hline$\varepsilon^{\alpha}$ & volume fraction of the phase $\alpha$ \\
\hline$\eta$ & viscosity parameter of the solid phase \\
\hline$\mu^{\alpha}$ & dynamic viscosity of the phase $\alpha$ \\
\hline$\rho^{\alpha}$ & density of the phase $\alpha$ \\
\hline$\sigma_{i j}$ & interfacial tension between fluid phases $i$ and $j$ \\
\hline$\psi^{\alpha}$ & adhesion of the phase $\alpha$ \\
\hline$\omega^{N \bar{t}}$ & mass fraction of necrotic cells in the tumor cells phase \\
\hline$\omega^{\overline{n l}}$ & nutrient mass fraction in the interstitial fluid \\
\hline$\omega_{c r i t}^{\overline{n l}}$ & critical nutrient mass fraction for growth \\
\hline$\omega_{e n v}^{\overline{n l}}$ & reference nutrient mass fraction in the environment \\
\hline$\stackrel{\kappa}{M}$ & inter-phase mass transfer \\
\hline$\varepsilon^{\alpha} r^{i \alpha}$ & reaction term i.e. intra-phase mass transfer. \\
\hline \multicolumn{2}{|c|}{ Subscripts and superscripts } \\
\hline crit & critical value for growth \\
\hline$n$ & nutrient \\
\hline$h$ & host cell phase \\
\hline$l$ & interstitial fluid \\
\hline$s$ & solid \\
\hline$t$ & tumor cell phase \\
\hline$\alpha$ & phase indicator with $\alpha=t, h, l$, or $s$ \\
\hline
\end{tabular}

Authors' contributions

BAS contributed the theoretical framework and helped in drafting the manuscript. RS developed the ECM deposition equations and implemented them in the code, performed all simulations and drafted the manuscript. PM developed and implemented in the code the equations for lysis. CS carried out the experimental data at HMRI. PD gave suggestions to clarify the work and MF contributed to the background of this work. All authors read and approved the final manuscript.

\footnotetext{
Author details

${ }^{1}$ Department of Civil, Environmental and Architectural Engineering, University of Padua, Padua 35131, Italy. ${ }^{2}$ Department of Translational Imaging, The Methodist Hospital Research Institute, Houston, USA. ${ }^{3}$ Department of Nanomedicine, The Methodist Hospital Research Institute, Houston, USA. ${ }^{4}$ Department of Medicine, Weill Cornell Medical College of Cornell University, New York, USA. ${ }^{5}$ Drug Discovery and Development Department, Fondazione Istituto Italiano di Tecnologia, Genoa 16163, Italy.
}

\section{Acknowledgements}

RS acknowledge the University of Padua for financial support (project n. CPDR121149). MF acknowledges the financial supports from NCI Physical Science-Oncology Centers (NIH U54CA143837), and from The Methodist Hospital Research Institute, including the Ernest Cockrell Jr. Presidential Distinguished Chair. PD acknowledges partial support from the European Research Council under the European Union's Seventh Framework Programme (FP7/2007-2013)/ERC Grant agreement No. 616695 and the Houston Methodist Research Institute.

Compliance with ethical guidelines

Competing interests

The authors declare that they have no competing interests.

\section{Appendix: Numerical solution and computational procedure}

The system of equations presented in the main text is solved by means of a partitioned approach within the framework of a staggered algorithm preserving the coupling nature 
of the multiphysics problem. The model is implemented in CAST3M (http://www. cast3m.cea.fr) where the mass balance equations are introduced in a slightly modified form according to the procedure of [22] which is here updated. More in detail, we call $\mathbf{d}_{s p}^{\overline{\bar{s}}}$ the elastic strain rate induced by the solid pressure

$$
\mathbf{d}_{s p}^{\overline{\bar{s}}}=\frac{\bar{\alpha}}{3 K} \frac{\partial p^{s}}{\partial t} \mathbf{1}
$$

where $\mathrm{K}$ is the bulk modulus of the unsaturated ECM scaffold. Introduction of the rate of the solid pressure, Eq. (14) yields

$$
\mathbf{1}: \mathbf{d}_{s p}^{\overline{\bar{s}}}=\frac{\bar{\alpha}}{K}\left(S^{t}+p^{t h} \frac{\partial S^{t}}{\partial p^{t h}}\right) \frac{\partial p^{t h}}{\partial t}+\frac{\bar{\alpha}}{K}\left(1-S^{l}-p^{h l} \frac{\partial S^{l}}{\partial p^{h l}}\right) \frac{\partial p^{h l}}{\partial t}+\frac{\bar{\alpha}}{K} \frac{\partial p^{l}}{\partial t}
$$

We consider now e.g. the mass balance equation of the tumor phase (Eq. (4)): by adding and subtracting $S^{t}\left(\mathbf{1}: \mathbf{d}_{s p}^{\overline{\bar{s}}}\right)$, moving the subtracted quantity to the lhs, and exploiting (27) this equation becomes

$$
\begin{aligned}
& {\left[\frac{\varepsilon S^{t}}{K_{t}}+\left(\frac{S^{t}(1-\varepsilon)}{K_{s}}+\frac{S^{t} \alpha}{K}\right)\left(S^{t}+p^{t h} \frac{\partial S^{t}}{\partial p^{t h}}\right)+\varepsilon \frac{\partial S^{t}}{\partial p^{t h}}\right] \frac{\partial p^{t h}}{\partial t}} \\
& +\left[\frac{\varepsilon S^{t}}{K_{t}}+\left(\frac{S^{t}(1-\varepsilon)}{K_{s}}+\frac{S^{t} \alpha}{K}\right)\left(1-S^{l}-p^{h l} \frac{\partial S^{l}}{\partial p^{h l}}\right)\right] \frac{\partial p^{h l}}{\partial t} \\
& +\left[\frac{\varepsilon S^{t}}{K_{t}}+\frac{S^{t}(1-\varepsilon)}{K_{s}}+\frac{S^{t} \alpha}{K}\right] \frac{\partial p^{l}}{\partial t} \\
& =\nabla \cdot\left[\frac{k_{r e l}^{t} \mathbf{k}}{\mu^{t}} \cdot \nabla\left(p^{l}+p^{h l}+p^{t h}\right)\right]-S^{t}\left[\mathbf{1}:\left(\mathbf{d}^{\overline{\bar{s}}}-\mathbf{d}_{s p}^{\overline{\bar{s}}}\right)\right] \\
& -\nabla S^{t} \cdot\left(\varepsilon \mathbf{v}^{\bar{s}}\right)+\frac{1}{\rho^{t}}\left(\begin{array}{c}
l \rightarrow t \\
\underset{\text { growth }}{M}-\stackrel{t \rightarrow l}{M}
\end{array}\right)+S_{\text {lysis }}^{t} \frac{\stackrel{l \rightarrow s}{M}}{\rho^{S}}
\end{aligned}
$$

The same is carried out for Eqs. $(5,7)$.

The weak form of Eqs. (4), (5), (7), (12) and (15) in their modified form as above is obtained by means of the standard Galerkin procedure and is then discretized in space by means of the Finite Element Method [32]. The primary variables are expressed in terms of their nodal values as

$$
\begin{aligned}
p^{t h}(t) \cong \mathbf{N}_{t} \overline{\mathbf{p}}^{t h}(t) p^{h l}(t) \cong \mathbf{N}_{h} \overline{\mathbf{p}}^{h l}(t) \quad p^{l}(t) \cong \mathbf{N}_{l} \overline{\mathbf{p}}^{l}(t) \\
\omega^{\overline{n l}}(t) \cong \mathbf{N}_{n} \overline{\boldsymbol{\omega}}^{\overline{n l}}(t) \quad \mathbf{u}^{s}(t) \cong \mathbf{N}_{u} \overline{\mathbf{u}}^{s}(t)
\end{aligned}
$$

where $\overline{\boldsymbol{\omega}}_{i}^{\overline{n l}}(t), \overline{\mathbf{p}}^{t h}(t), \overline{\mathbf{p}}^{h l}(t), \overline{\mathbf{p}}^{l}(t), \overline{\mathbf{u}}^{s}(t)$ are vectors of nodal values of the primary variables at time instant $\mathbf{t}$, and $\mathbf{N}_{n}, \mathbf{N}_{t}, \mathbf{N}_{h}, \mathbf{N}_{l}$, and $\mathbf{N}_{u}$ are vectors/matrices of shape functions related to these variables.

Integration in the time domain is carried out by the Finite Difference Method adopting a quasi-Crank-Nicolson scheme $(\theta$-Wilson method with $\theta=0.52)$. Within each time step the equations are linearized by the Newton-Raphson method. For the numerical solution of the resulting system of equations, a staggered scheme is adopted with 
iterations within each time step to preserve the coupled nature of the system. The convergence properties of such staggered schemes have been investigated by Turska and Schrefler [50]. In particular, for the iteration convergence within each computational step a lower limit of $\Delta \mathrm{t} / \mathrm{h}^{2}$, function of the material properties, has to be observed; $\Delta \mathrm{t}$ is the time step and $h$ the element size. The existence of this limit means that we cannot diminish the time step at will below a certain threshold without also decreasing the element size. For the simulations in this paper we choose $\Delta t=6 \mathrm{~min}$ and the limit $\Delta t /$ $\mathrm{h}^{2}=3 \cdot 10^{13}$.

Three computational units are used in the staggered scheme: the first is for the nutrient mass fraction $\omega^{\overline{n l}}$, the second to compute pth, phl and $\mathrm{pl}$, and the third is used to obtain the displacement vector us. Within each iteration the mass fraction of NTC, $\omega^{N \bar{t}}$, is updated using Eq. (11).

Taking into account the chosen staggered scheme, the final system of equations can be expressed in matrix form as follows, where some of the coupling terms have been placed in the source terms and are updated at each iteration

$$
\mathbf{C}_{i j}(\mathbf{x}) \frac{\partial \mathbf{x}}{\partial t}+\mathbf{K}_{i j}(\mathbf{x}) \mathbf{x}=\mathbf{f}_{i}(\mathbf{x})
$$

with

$$
\mathbf{C}_{i j}=\left(\begin{array}{ccccc}
\mathbf{C}_{n n} & 0 & 0 & 0 & 0 \\
0 & \mathbf{C}_{t t} & \mathbf{C}_{t h} & \mathbf{C}_{t l} & 0 \\
0 & \mathbf{C}_{h t} & \mathbf{C}_{h h} & \mathbf{C}_{t l} & 0 \\
0 & \mathbf{C}_{l t} & \mathbf{C}_{l h} & \mathbf{C}_{l l} & 0 \\
0 & 0 & 0 & 0 & \mathbf{C}_{u u}
\end{array}\right) \quad \mathbf{K}_{i j}=\left(\begin{array}{ccccc}
\mathbf{K}_{n n} & 0 & 0 & 0 & 0 \\
0 & \mathbf{K}_{t t} & \mathbf{K}_{t h} & \mathbf{K}_{t l} & 0 \\
0 & \mathbf{K}_{h t} & \mathbf{K}_{h h} & \mathbf{K}_{h l} & 0 \\
0 & \mathbf{K}_{l t} & \mathbf{K}_{l h} & \mathbf{K}_{l l} & 0 \\
0 & 0 & 0 & 0 & 0
\end{array}\right), \mathbf{f}_{i}=\left(\begin{array}{c}
\mathbf{f}_{n} \\
\mathbf{f}_{t} \\
\mathbf{f}_{h} \\
\mathbf{f}_{l} \\
\mathbf{f}_{u}
\end{array}\right)
$$

where $\mathbf{x}^{T}=\left\{\bar{\omega}^{\overline{n l}}, \overline{\mathbf{p}}^{t h}, \overline{\mathbf{p}}^{h l}, \overline{\mathbf{p}}^{l}, \overline{\mathbf{u}}^{\mathbf{s}}\right\}$.

The modular computational structure allows more than one chemical species to be taken into account, simply by adding a computational unit (equivalent to the first one used for the nutrient) for each of the additional chemical species considered

The nonlinear coefficient matrices $\mathbf{C}_{i j}(\mathbf{x}), \mathbf{K}_{i j}(\mathbf{x})$ and $\mathbf{f}_{i}(\mathbf{x})$ are given below.

$$
\begin{aligned}
& \mathbf{C}_{n n}=\int_{\Omega} \mathbf{N}_{n}^{T}\left(\varepsilon S^{l} \mathbf{N}_{n}\right) d \Omega \\
& \mathbf{C}_{t t}=\int_{\Omega} \mathbf{N}_{t}^{T}\left[\frac{\varepsilon S^{t}}{K_{t}} \mathbf{N}_{t}+\left(\frac{S^{t}(1-\varepsilon)}{K_{s}}+\frac{S^{t} \alpha}{K}\right)\left(S^{t}+p^{t h} \frac{\partial S^{t}}{\partial p^{t h}}\right) \mathbf{N}_{t}+\varepsilon \frac{\partial S^{t}}{\partial p^{t h}} \mathbf{N}_{t}\right] d \Omega \\
& \mathbf{C}_{t h}=\int_{\Omega} \mathbf{N}_{t}^{T}\left[\frac{\varepsilon S^{t}}{K_{t}} \mathbf{N}_{h}+\left(\frac{S^{t}(1-\varepsilon)}{K_{s}}+\frac{S^{t} \alpha}{K}\right)\left(1-S^{l}-p^{h l} \frac{\partial S^{l}}{\partial p^{h l}}\right) \mathbf{N}_{h}\right] d \Omega \\
& \mathbf{C}_{t l}=\int_{\Omega} \mathbf{N}_{t}^{T}\left(\frac{\varepsilon S^{t}}{K_{t}} \mathbf{N}_{l}+\frac{S^{t}(1-\varepsilon)}{K_{s}} \mathbf{N}_{l}+\frac{S^{t} \alpha}{K} \mathbf{N}_{l}\right) d \Omega
\end{aligned}
$$




$$
\begin{aligned}
& \mathbf{C}_{h t}=\int_{\Omega} \mathbf{N}_{h}^{T}\left[\left(\frac{S^{h}(1-\varepsilon)}{K_{s}}+\frac{S^{h} \alpha}{K}\right)\left(S^{t}+p^{t h} \frac{\partial S^{t}}{\partial p^{t h}}\right) \mathbf{N}_{t}-\varepsilon \frac{\partial S^{t}}{\partial p^{t h}} \mathbf{N}_{t}\right] d \Omega \\
& \mathbf{C}_{h h}=\int_{\Omega} \mathbf{N}_{h}^{T}\left[\frac{\varepsilon S^{h}}{K_{h}} \mathbf{N}_{h}+\left(\frac{S^{h}(1-\varepsilon)}{K_{s}}+\frac{S^{h} \alpha}{K}\right)\left(1-S^{l}-p^{h l} \frac{\partial S^{l}}{\partial p^{h l}}\right) \mathbf{N}_{h}-\varepsilon \frac{\partial S^{l}}{\partial p^{h l}} \mathbf{N}_{h}\right] d \Omega \\
& \mathbf{C}_{h l}=\int_{\Omega} \mathbf{N}_{h}^{T}\left(\frac{\varepsilon S^{h}}{K_{h}} \mathbf{N}_{l}+\frac{S^{h}(1-\varepsilon)}{K_{s}} \mathbf{N}_{l}+\frac{S^{h} \alpha}{K} \mathbf{N}_{l}\right) d \Omega \\
& \mathbf{C}_{l t}=\int_{\Omega} \mathbf{N}_{l}^{T}\left[\frac{\varepsilon S^{t}}{K_{t}} \mathbf{N}_{t}+\left(\frac{1-\varepsilon}{K_{s}}+\frac{\alpha}{K}\right)\left(S^{t}+p^{t h} \frac{\partial S^{t}}{\partial p^{t h}}\right) \mathbf{N}_{t}\right] d \Omega \\
& \mathbf{C}_{l h}=\int_{\Omega} \mathbf{N}_{l}^{T}\left[\frac{\varepsilon S^{t}}{K_{t}} \mathbf{N}_{h}+\frac{\varepsilon S^{h}}{K_{h}} \mathbf{N}_{h}+\left(\frac{1-\varepsilon}{K_{s}}+\frac{\alpha}{K}\right)\left(1-S^{l}-p^{h l} \frac{\partial S^{l}}{\partial p^{h l}}\right) \mathbf{N}_{h}\right] d \Omega \\
& \mathbf{C}_{l l}=\int_{\Omega} \mathbf{N}_{l}^{T}\left(\frac{\varepsilon S^{t}}{K_{t}} \mathbf{N}_{l}+\frac{\varepsilon S^{h}}{K_{h}} \mathbf{N}_{l}+\frac{\varepsilon S^{l}}{K_{l}} \mathbf{N}_{l}+\frac{1-\varepsilon}{K_{s}} \mathbf{N}_{l}+\frac{\alpha}{K} \mathbf{N}_{l}\right) d \Omega \\
& \left(\mathbf{C}_{u u}\right)_{i j}=-\int_{\Omega} \mathbf{B}^{\mathrm{T}} \mathbf{D}_{s} \mathbf{B} d \Omega \\
& \mathbf{K}_{n n}=\int_{\Omega}\left(\nabla \mathbf{N}_{n}\right)^{\mathrm{T}}\left(\varepsilon S^{l} D_{e f f}^{\overline{n l}} \nabla \mathbf{N}_{n}\right) d \Omega \\
& \mathbf{K}_{t t}=\int_{\Omega}\left(\nabla \mathbf{N}_{t}\right)^{\mathrm{T}}\left(\frac{k_{r e l}^{t} \mathbf{k}}{\mu^{t}} \nabla \mathbf{N}_{t}\right) d \Omega \\
& \mathbf{K}_{t h}=\int_{\Omega}\left(\nabla \mathbf{N}_{t}\right)^{\mathrm{T}}\left(\frac{k_{r e l}^{t} \mathbf{k}}{\mu^{t}} \nabla \mathbf{N}_{h}\right) d \Omega
\end{aligned}
$$




$$
\mathbf{K}_{t l}=\int_{\Omega}\left(\nabla \mathbf{N}_{t}\right)^{\mathrm{T}}\left(\frac{k_{r e l}^{t} \mathbf{k}}{\mu^{t}} \nabla \mathbf{N}_{l}\right) d \Omega
$$

$$
\mathbf{K}_{h t}=0
$$

$$
\mathbf{K}_{h h}=\int_{\Omega}\left(\nabla \mathbf{N}_{h}\right)^{\mathrm{T}}\left(\frac{k_{r e l}^{h} \mathbf{k}}{\mu^{h}} \nabla \mathbf{N}_{h}\right) d \Omega
$$

$$
\mathbf{K}_{h l}=\int_{\Omega}\left(\nabla \mathbf{N}_{h}\right)^{\mathrm{T}}\left(\frac{k_{r e l}^{h} \mathbf{k}}{\mu^{h}} \nabla \mathbf{N}_{l}\right) d \Omega
$$

$$
\mathbf{K}_{l t}=\int_{\Omega}\left(\nabla \mathbf{N}_{l}\right)^{\mathrm{T}}\left(\frac{k_{r e l}^{t} \mathbf{k}}{\mu^{t}} \nabla \mathbf{N}_{t}\right) d \Omega
$$

$$
\mathbf{K}_{l h}=\int_{\Omega}\left(\nabla \mathbf{N}_{l}\right)^{\mathrm{T}}\left(\frac{k_{r e l}^{t} \mathbf{k}}{\mu^{t}} \nabla \mathbf{N}_{h}+\frac{k_{r e l}^{h} \mathbf{k}}{\mu^{h}} \nabla \mathbf{N}_{h}\right) d \Omega
$$

$$
\mathbf{K}_{l l}=\int_{\Omega}\left(\nabla \mathbf{N}_{l}\right)^{\mathrm{T}}\left(\frac{k_{r e l}^{t} \mathbf{k}}{\mu^{t}} \nabla \mathbf{N}_{l}+\frac{k_{r e l}^{h} \mathbf{k}}{\mu^{h}} \nabla \mathbf{N}_{l}+\frac{k_{r e l}^{l} \mathbf{k}}{\mu^{l}} \nabla \mathbf{N}_{l}\right) d \Omega
$$

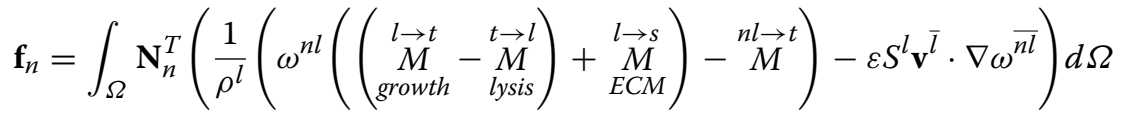

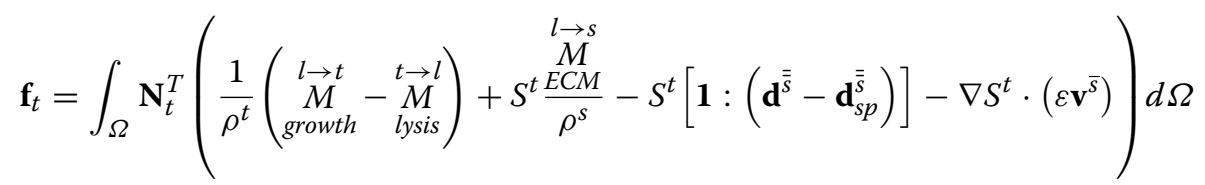

$$
\begin{aligned}
& \mathbf{f}_{h}=\int_{\Omega} \mathbf{N}_{h}^{T}\left(\begin{array}{c}
l \rightarrow s \\
S^{h} \frac{E C M}{\rho^{s}}
\end{array}-S^{h}\left[\mathbf{1}:\left(\mathbf{d}^{\overline{\bar{s}}}-\mathbf{d}_{s p}^{\overline{\bar{s}}}\right)\right]-\nabla S^{h} \cdot\left(\varepsilon \mathbf{v}^{\bar{s}}\right)\right) d \Omega
\end{aligned}
$$




$$
\begin{aligned}
& \mathbf{f}_{l}=\int_{\Omega} \mathbf{N}_{l}^{T}\left(-\mathbf{1}:\left(\mathbf{d}^{\overline{\bar{s}}}-\mathbf{d}_{s p}^{\overline{\bar{s}}}\right)+\frac{\rho^{l}-\rho^{t}}{\rho^{t} \rho^{l}}\left(\underset{\text { growth }}{\stackrel{M_{\text {lysis }}^{t}}{M}} \stackrel{\stackrel{t \rightarrow l}{M}}{\rho^{l}}\right)+\frac{\rho^{l}-\rho^{s}}{\rho^{s} \rho^{l}} \underset{E C M}{\stackrel{l}{M}}\right) d \Omega \\
& \mathbf{f}_{u}=\int_{\Omega} \mathbf{B}^{T}\left(\mathbf{D}_{s} \mathbf{d}_{v p}^{\overline{\bar{s}}}\right) d \Omega+\int_{\Omega} \mathbf{B}^{T}\left(\mathbf{D}_{s} \mathbf{d}_{s p}^{\overline{\bar{s}}}\right) d \Omega
\end{aligned}
$$

Received: 14 May 2015 Accepted: 22 July 2015

Published online: 08 August 2015

\section{References}

1. Roose T, Chapman SJ, Maini PK (2007) Mathematical models of avascular tumor growth. Siam Rev 49(2):179-208

2. Sciumè G, Gray W, Ferrari M, Decuzzi P, Schrefler B (2013) On computational modeling in tumor growth. Arch Comput Methods Eng 20:327-352

3. Deisboeck TS, Wang Z, Macklin P, Cristini V (2011) Multiscale cancer modeling. Annu Rev Biomed Eng 13:127-155. doi:10.1146/annurev-bioeng-071910-124729

4. Wise SM, Lowengrub JS, Frieboes HB, Cristini V (2008) Three-dimensional multispecies nonlinear tumor growth—I: model and numerical method. J Theor Biol 253:524-543

5. Frieboes HB, Edgerton ME, Fruehauf JP, Rose FR, Worrall LK, Gatenby RA et al (2009) Prediction of drug response in breast cancer using integrative experimental/computational modeling. Cancer Res 69:4484-4492

6. Macklin P, McDougall S, Anderson AR, Chaplain MA, Cristini V, Lowengrub J (2009) Multiscale modelling and nonlinear simulation of vascular tumour growth. J Math Biol 58:765-798

7. Lowengrub JS, Frieboes HB, Jin F, Chuang Y, Li X, Macklin P et al (2010) Nonlinear modelling of cancer: bridging the gap between cells and tumours. Nonlinearity 23:R1. doi:10.1088/0951-7715/23/1/R01

8. Bearer EL, Lowengrub JS, Frieboes HB, Chuang Y-L, Jin F, Wise SM et al (2009) Multiparameter computational modeling of tumor invasion. Cancer Res 69:4493-4501

9. Hawkins-Daarud A, van der Zee KG, Tinsley Oden J (2012) Numerical simulation of a thermodynamically consistent four-species tumor growth model. Int J Numer Methods in Biomed Eng 28:3-24

10. Narayanan H, Verner S, Mills K, Kemkemer R, Garikipati K (2010) In silico estimates of the free energy rates in growing tumor spheroids. J Phys Condens Matter Special Issue on Cell-Substrate Interact 22:194122

11. Netti P, Berk D, Swartz M, Grodzinsky A, Jain R (2000) Role of extracellular matrix assembly in interstitial transport in solid tumors. Cancer Res 60:2497-2503

12. Jain RK (1999) Transport of molecules, particles, and cells in solid tumors. Annu Rev Biomed Eng 1:241-263

13. Jain RK, Stylianopoulos T (2010) Delivering nanomedicine to solid tumors. Nature Rev Clin Oncol 7:653-664

14. Sciumè G, Shelton S, Gray W, Miller C, Hussain F, Ferrari M et al (2012) Tumor growth modeling from the perspective of multiphase porous media mechanics. Mol Cell Biomech MCB 9(3):193

15. Sciumè G, Shelton S, Gray W, Miller C, Hussain F, Ferrari M et al (2013) A multiphase model for three-dimensional tumor growth. New J Phys 15(1):015005

16. Bao G, Bazilevs Y, Chung J-H, Decuzzi P, Espinosa HD, Ferrari M et al (2014) USNCTAM perspectives on mechanics in medicine. J Royal Soc Interface 11(97):20140301

17. Sciumè G, Gray W, Hussain F, Ferrari M, Decuzzi P, Schrefler BA (2014) Three phase flow dynamics in tumor growth. Comput Mech 53:465-484

18. Sciumè G, Ferrari M, Schrefler BA (2014) Saturation-pressure relationships for two-and three-phase flow analogies for soft matter. Mech Res Commun 62:132-137

19. Yuhas JM, Li AP, Martinez AO, Ladman AJ (1977) A simplified method for production and growth of multicellular tumor spheroids. Cancer Res 37:3639-3643

20. Chignola R, Foroni R, Franceschi A, Pasti M, Candiani C, Anselmi C et al (1995) Heterogeneous response of individual multicellular tumour spheroids to immunotoxins and ricin toxin. Br J Cancer 72:607-614

21. Chignola R, Schenetti A, Andrighetto G, Chiesa E, Foroni R, Sartoris S et al (2000) Forecasting the growth of multicell tumour spheroids: implications for the dynamic growth of solid tumours. Cell Prolif 33:219-229

22. Sciumè G, Santagiuliana R, Ferrari M, Decuzzi P, Schrefler B (2014) A tumor growth model with deformable ECM. Phys Biol 11:065004. doi:10.1088/1478-3975/11/6/065004

23. Chung LS, Y-g Man, Lupton GP (2010) WT-1 expression in a spectrum of melanocytic lesions: implication for differential diagnosis. J Cancer 1:120-125. doi:10.7150/jca.1.120

24. Gray WG, Miller TC (2014) Introduction to the thermodynamically constrained averaging theory for porous medium systems. Springer, Switzerland

25. Gray WG, Miller CT, Schrefler BA (2013) Averaging theory for description of environmental problems: what have we learned. Adv Water Resour 51:123-138

26. Gray WG, Schrefler BA (2007) Analysis of the solid phase stress tensor in multiphase porous media. Int J Numer Anal Meth Geomech 31(4):541-581 
27. Helmlinger G, Netti PA, Lichtenbeld HC, Melder RJ, Jain RK (1997) Solid stress inhibits the growth of multicellular tumor spheroids. Nat Biotechnol 15(8):778-783

28. Meroi E, Schrefler BA, Zienkiewicz O (1995) Large strain static and dynamic semisaturated soil behaviour. Int J Numer Anal Meth Geomech 19(2):81-106

29. Bažant ZP, Gattu M, Vorel J (2012) Work conjugacy error in commercial finite-element codes: its magnitude and how to compensate for it. Proc R Soc A Math Phys Eng Sci 468(2146):3047-3058

30. Cheng YM, Tsui Y (1992) Limitations to the large strain theory. Int J Numer Meth Eng 33(1):101-114

31. Preziosi L, Ambrosi D, Verdier C (2010) An elasto-visco-plastic model of cell aggregates. J Theor Biol 262(1):35-47

32. Lewis RW, Schrefler BA (1998) The finite element method in the static and dynamic deformation and consolidation of porous media. Wiley, Chichester

33. Sciumè G, Benboudjema F, De Sa C, Pesavento F, Berthaud Y, Schrefler BA (2013) A multiphysics model for concrete at early age applied to repairs problems. Eng Struct 57:374-387

34. Zienkiewicz OC, Taylor RL (2000) The finite element method: solid mechanics. Butterworth-heinemann, Oxford

35. Simo J, Hughes T (2008) Computational inelasticity. Springer, New York

36. Sutherland RM (1988) Cell and environment interactions in tumor microregions: the multicell spheroid model. Science NY 240(4849):177-184

37. Frieboes HB, Zheng X, Sun CH, Tromberg B, Gatenby R, Cristini V (2006) An integrated computational/experimental model of tumor invasion. Cancer Res 66(3):1597-1604. doi:10.1158/0008-5472.CAN-05-3166

38. Carlsson J, Yuhas JM (1984) Liquid-overlay culture of cellular spheroids. Recent Results in Cancer Research. Fortschritte Der Krebsforschung. Progres Dans Les Recherches Sur Le Cancer 95(Foa 4):1-23

39. Byrne H, Preziosi L (2003) Modelling solid tumour growth using the theory of mixtures. Math Med Biol 20(4):341-366

40. Ambrosi D, Mollica F (2004) The role of stress in the growth of a multicell spheroid. J Math Biol 499:477-499

41. Ciarletta P, Ambrosi D, Maugin GA, Preziosi L (2013) Mechano-transduction in tumour growth modelling. Eur Phys J E 36(3):23. doi:10.1140/epje/i2013-13023-2

42. Wise SM, Lowengrub JS, Frieboes HB, Cristini V (2008) Three-dimensional multispecies nonlinear tumor growth-I Model and numerical method. J Theor Biol 253(3):524-543. doi:10.1016/j.jtbi.2008.03.027

43. Mishra DK, Sakamoto JH, Thrall MJ, Baird BN, Blackmon SH, Ferrari M et al (2012) Human lung cancer cells grown in an ex vivo 3D lung model produce matrix metalloproteinases not produced in 2D culture. PLoS One 7(9):e45308

44. Walenta S, Doetsch J, Mueller-Klieser W, Kunz-Schughart LA (2000) Metabolic imaging in multicellular spheroids of oncogene-transfected fibroblasts. J Histochem Cytochem 48(4):509-522

45. Mueller-Klieser W, Freyer J, Sutherland R (1986) Influence of glucose and oxygen supply conditions on the oxygenation of multicellular spheroids. Br J Cancer 53(3):345-353

46. Galbusera F, Cioffi M, Raimondi MT (2008) An in silico bioreactor for simulating laboratory experiments in tissue engineering. Biomed Microdevices 10(4):547-554

47. Ambrosi D, Preziosi L, Vitale G (2012) The interplay between stress and growth in solid tumors. Mech Res Commun 42:87-91

48. Forgacs G, Foty RA, Shafrir Y, Steinberg MS (1998) Viscoelastic properties of living embryonic tissues: a quantitative study. Biophys J 74(5):2227-2234

49. Cristini V, Lowengrub J (2010) Multiscale modeling of cancer. Cambridge University Press, Cambridge

50. Turska E, Schrefler BA (1993) On convergence conditions of partitioned solution procedures for consolidation problems. Comput Methods Appl Mech Eng 106(1-2):51-63

\section{Submit your manuscript to a SpringerOpen ${ }^{\circ}$ journal and benefit from:}

- Convenient online submission

- Rigorous peer review

- Immediate publication on acceptance

- Open access: articles freely available online

- High visibility within the field

- Retaining the copyright to your article

Submit your next manuscript at $\mathbf{s p r i n g e r o p e n . c o m ~}$ 